\title{
Asymptotic Efficiency of the Blest Type Tests of Independence
}

by

\section{Shu Wang}

\author{
A Thesis submitted to \\ the Faculty of Graduate Studies and Research \\ in partial fulfilment of \\ the requirements for the degree of
}

Master of Science

Ottawa-Carleton Institute for

Mathematics and Statistics

School of Mathematics and Statistics

Carleton University

Ottawa, Ontario, Canada

November 2006

Copyright (C)

2006 - Shu Wang 


$\begin{array}{ll}\begin{array}{l}\text { Library and } \\ \text { Archives Canada }\end{array} & \begin{array}{l}\text { Bibliothèque et } \\ \text { Archives Canada }\end{array} \\ \begin{array}{l}\text { Published Heritage } \\ \text { Branch }\end{array} & \begin{array}{l}\text { Direction du } \\ \text { Patrimoine de l'édition }\end{array} \\ \begin{array}{l}\text { 395 Wellington Street } \\ \text { Ottawa ON K1A ON4 }\end{array} & \begin{array}{l}\text { 395, rue Wellington } \\ \text { Ottawa ON K1A ON4 } \\ \text { Canada }\end{array}\end{array}$

Your file Votre référence ISBN: 978-0-494-33715-8 Our file Notre référence ISBN: 978-0-494-33715-8

NOTICE:

The author has granted a nonexclusive license allowing Library and Archives Canada to reproduce, publish, archive, preserve, conserve, communicate to the public by telecommunication or on the Internet, loan, distribute and sell theses worldwide, for commercial or noncommercial purposes, in microform, paper, electronic and/or any other formats.

The author retains copyright ownership and moral rights in this thesis. Neither the thesis nor substantial extracts from it may be printed or otherwise reproduced without the author's permission.
AVIS:

L'auteur a accordé une licence non exclusive permettant à la Bibliothèque et Archives Canada de reproduire, publier, archiver, sauvegarder, conserver, transmettre au public par télécommunication ou par l'Internet, prêter, distribuer et vendre des thèses partout dans le monde, à des fins commerciales ou autres, sur support microforme, papier, électronique et/ou autres formats.

L'auteur conserve la propriété du droit d'auteur et des droits moraux qui protège cette thèse. $\mathrm{Ni}$ la thèse ni des extraits substantiels de celle-ci ne doivent être imprimés ou autrement reproduits sans son autorisation.
In compliance with the Canadian

Privacy Act some supporting forms may have been removed from this thesis.

While these forms may be included in the document page count, their removal does not represent any loss of content from the thesis.
Conformément à la loi canadienne sur la protection de la vie privée, quelques formulaires secondaires ont été enlevés de cette thèse.

Bien que ces formulaires aient inclus dans la pagination, il n'y aura aucun contenu manquant.

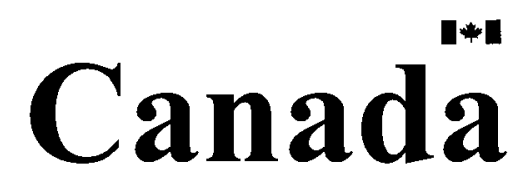




\section{Abstract}

In 2000 Blest proposed a new measure of rank correlation which is sensitive to discrepancies in the small ranks. This measure can be used to compare various methods of data analysis in computer modeling and to evaluate the prediction accuracy. Blest's statistic, however, has a defect of being dissymmetric in its arguments. The modification of Blest's statistic proposed by Genest and Plante in 2003 eliminates the dissymmetry. In this project, efficiency properties of nonparametric tests for independence based on Blest's correlation coefficient and its symmetric modification are investigated. Pitman efficiencies of the Blest type tests for a wide class of alternatives close to the null hypothesis of independence are calculated. Pitman efficiency comparisons are made with analogous tests existing in the literature. Conditions for Pitman optimality of the Blest type tests are established. 


\section{Acknowledgments}

I would like to first extend my sincere appreciation to my supervisor, Dr. Natalia Stepanova, for her guidance and support throughout my time in the Master of Science program at Carleton University. This project would not have been possible without her inspiration and help. I would also like to thank Drs. Raluca Balan, Evangelos Kranakis and Majid Mojirsheibani for serving on my graduate committee.

Finally I would like to thank my husband, Wei, my son, Jeff, and my mom and dad, Guozheng and Yongzhong, for their love and support during the time I have spent at Carleton University. 


\section{Table of Contents}

Abstract

Acknowledgments

Table of Contents $\quad$ iv

List of Tables $\quad$ vi

$\begin{array}{ll}\text { List of Figures } & \text { vii }\end{array}$

1 Introduction $\quad 1$

2 Formulation of the Problem and Notation 4

3 Rank Statistics for Independence $\quad 9$

3.1 Linear Rank Statistics . . . . . . . . . . . . . . . . . . . 9 9

3.2 Asymptotic Normality of Linear Rank Statistics . . . . . . . . . . . . . 11

4 Blest's Measure of Rank Correlation $\quad 13$

4.1 Blest's Measure of Rank Correlation . . . . . . . . . . . . . . . . . . 13

4.2 Symmetrized Version of Blest's Statistics . . . . . . . . . . . . 16

5 Asymptotic Relative Efficiency of the Blest Type Tests 18

5.1 Concept of Asymptotic Relative Efficiency . . . . . . . . . . . . . 18

5.2 Asymptotic Normality of the Blest Type Tests . . . . . . . . . . . . 21

5.3 Local Limiting Power Functions of the Blest Type Tests . . . . . . . . . . . 27 


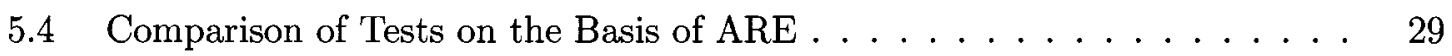

6 Upper Bound on the Power Function 36

6.1 Concept of Local Asymptotic Normality . . . . . . . . . . . . . . 37

6.2 LAN of the Underlying Family of Distributions . . . . . . . . . . . . 39

6.3 Pitman Absolute Efficiency of the Blest Type Tests . . . . . . . . . . . . . 39

7 Local Asymptotic Optimality of the Blest Type Tests 40

8 Conclusion $\quad 45$

Appendix A Calculation of Relative Efficiency $\quad 46$

$\begin{array}{ll}\text { Bibliography } & \mathbf{5 2}\end{array}$ 


\section{List of Tables}

1 Rankings correspond to sensitivity techniques $A$ and $B \ldots \ldots 2$

2 The accumulated ranks and their differences, for $n=6$ and $k=1, \ldots, 6 \ldots 14$

3 Relative efficiencies of the Blest type test statistics versus the top-down test statistic for the Woodworth distribution . . . . . . . . . . . . . 31

4 Relative efficiencies of the symmetrized Blest test statistics versus the weighted Spearman's and Kendall's test statistics for the Woodworth dis-

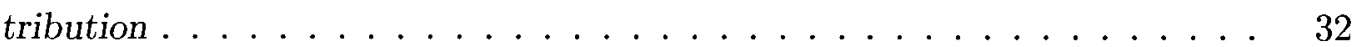

$5 \quad$ Relative efficiencies of the Blest type test statistics versus the top-down test statistic for the normal distribution . . . . . . . . . . . . 33

6 Relative efficiencies of the Blest type test statistics versus the weighted Spearman's and Kendall's test statistics for the normal distribution . . . . . . . .

$7 \quad$ Relative efficiencies of the Blest type test statistics versus the top-down test statistic in Example $3 \ldots \ldots \ldots \ldots \ldots$

8 Relative efficiencies of the Blest type test statistics versus the weighted Spearman's and Kendall's test statistics in Example $3 \ldots \ldots \ldots$. . . . . . 


\section{List of Figures}

1 Polygonal areas determined by cumulative ranks for $n=6$ and $k=1, \ldots, 6 \quad 15$

2 Polygonal areas, revised definition, for $n=6$ and $k=1, \ldots, 6 \ldots 16$ 


\section{Chapter 1}

\section{Introduction}

Testing for independence is a fundamental statistical problem with a long history. For testing independence of two random variable tests based on rank correlation coefficients are often used. Two of the rank correlation coefficients have been adopted widely: Spearman's $\rho$ and Kendall's $\tau$. The first statistic appeared in 1904 in the paper of psychologist C. Spearman [20]; the second one was introduced by statistician M. G. Kendall [11] in 1938. Let $\left(X_{i}, Y_{i}\right), i=1, \ldots, n$, be a random sample from a continuous bivariate distribution, and let $R_{i}$ and $Q_{i}$ denote the ranks of the $i$ th member in the $X$ - and $Y$-sample, respectively. Spearman's $\rho$ and Kendall's $\tau$ can be written as follows:

$$
\begin{gathered}
\rho_{n}=1-\frac{6}{n^{3}-n} \sum_{i=1}^{n}\left(Q_{i}-R_{i}\right)^{2}, \\
\tau_{n}=\frac{2}{n(n-1)} \sum_{1 \leq i<j \leq n} \operatorname{sign}\left(R_{i}-R_{j}\right) \operatorname{sign}\left(Q_{i}-Q_{j}\right),
\end{gathered}
$$

where $\operatorname{sign}(x)=1,0,-1$ if $x>0,=0,<0$, respectively. They are normalized to take the values between -1 and 1 ; and to be close to zero under the hypothesis of independence. Suppose that $R_{i}=i, i=1, \ldots, n$. Then Kendall's $\tau$ counts the minimum number of transpositions required to transform the permutation of the integers $\left(Q_{1}, Q_{2}, \ldots, Q_{n}\right)$ into natural order. Compared with Kendall's $\tau$, Spearman's $\rho$ gives greater weighting to differences between individual scorings, but both statistics are equally influenced by disagreement on the assignment of rankings at all levels. Therefore they do not work properly in the situations, in which agreement in the top rankings is of interest. For example, in evaluating 
Table 1: Rankings correspond to sensitivity techniques $A$ and $B$

\begin{tabular}{|c|c|c|c|c|c|c|c|c|c|c|}
\hline A & 1 & 2 & 3 & 4 & 5 & 6 & 7 & 8 & 9 & 10 \\
\hline B & 1 & 3 & 2 & 4 & 16 & 10 & 19 & 12 & 18 & 17 \\
\hline \hline A & 11 & 12 & 13 & 14 & 15 & 16 & 17 & 18 & 19 & 20 \\
\hline B & 20 & 5 & 14 & 7 & 8 & 11 & 6 & 15 & 9 & 13 \\
\hline
\end{tabular}

the prediction accuracy in subjectively judged sporting events such as ice skating, diving, gymnastics, etc. As an illustration, consider the experiment, in which 20 input variables are analyzed by two different sensitivity techniques, $A$ and $B$, see [9]. The rankings given by $A$ and $B$ are listed in Table 1 .

Although these two methods agree on the importance of the top four input variables, Spearman's $\rho$ is nonsignificant 0.2767 ( $p$-value $=0.1130$ ). Clearly, this statistic does not convey the information that the two methods agree on the four most influential inputs.

To deal with such situations, in 1987 Iman and Conover [9] proposed a so-called "topdown" correlation coefficient, which centers on agreement in the top rankings. It is defined by the formula

$$
r_{n}=\frac{1}{n-\phi(1)}\left(\sum_{i=1}^{n} \phi\left(R_{i}\right) \phi\left(Q_{i}\right)-n\right)
$$

where $\phi(i)=\sum_{j=i}^{n}(1 / j)$ are the Savage scores. Later on, Blest [1] introduced a new measure of association, which has greater power of discrimination between individual permutations than either Spearman's or Kendall's coefficients and is highly correlated with both of them. Blest's coefficient is defined by

$$
\nu_{n}=\frac{2 n+1}{n-1}-\frac{12}{n^{2}-n} \sum_{i=1}^{n}\left(1-\frac{R_{i}}{n+1}\right)^{2} Q_{i} .
$$

The statistic $\nu_{n}$ has a defect of being dissymmetric in its arguments. To eliminate the dissymmetry, Genest and Plante [5] proposed a symmetrized version of Blest's coefficient

$$
s_{n}=-\frac{4 n+5}{n-1}+\frac{6}{n^{3}-n} \sum_{i=1}^{n} R_{i} Q_{i}\left(4-\frac{R_{i}+Q_{i}}{n+1}\right),
$$


which retains the character of Blest's coefficient and, as we will show below, has often a better performance than the original one. Like the top-down correlation coefficient $r_{n}$, the statistics $\nu_{n}$ and $s_{n}$ can be used to compare various methods of data analysis in computer modeling and to evaluate the prediction accuracy. According to Genest and Plante [5], the Blest asymmetric measure of discrepancy $\nu_{n}$ is inappropriate as a test statistics for testing independence. Nevertheless, this statistic can yet safely be used for testing independence in case of (approximately) symmetric bivariate distribution. For this reason, efficiency properties of the tests based on Blest's coefficient $\nu_{n}$ are also of interest.

Test statistics that depend on the observations only through their ranks are attractive, because they are distribution-free under the null hypothesis and need not be less efficient, asymptotically. An important characteristic of nonparametric tests based on distributionfree statistics is their asymptotic relative efficiency (ARE). The concept of ARE allows us to compare tests available for testing the null hypothesis and to select the best one. In this project, we use the Pitman ARE [7], [21], to study the Blest type correlation coefficients. 


\section{Chapter 2}

\section{Formulation of the Problem and Notation}

Let $\left(X_{i}, Y_{i}\right), i=1, \ldots, n$, be a random sample from a continuous distribution with distribution function (d.f.) $H$ and marginal d.f.'s $F$ and $G$. Denote by $R_{i}$ the rank of $X_{i}$ among $X_{1}, \ldots, X_{n}$ and by $Q_{i}$ the rank of $Y_{i}$ among $Y_{1}, \ldots, Y_{n}$. We assume that the d.f. $H(x, y)=H_{\theta}(x, y)$ has the form

$$
H_{\theta}(x, y)=F(x) G(y)+\theta \Omega(F(x), G(y)), \quad(x, y) \in \mathbb{R}^{2},
$$

where $\theta$ is a parameter of association close to zero and $\Omega$ is the dependence function defined on the unit cube $I^{2}=[0,1] \times[0,1]$ that satisfies certain boundary conditions. We are interested in testing the hypothesis

$$
H_{0}: \theta=0
$$

against the one-sided alternatives

$$
H_{1}: \theta>0 \text { or } H_{1}^{\prime}: \theta<0
$$

Recall that the property of independence preserves under (measurable) transformations of random variables. Note also that $F\left(X_{i}\right)$ and $G\left(Y_{i}\right), i=1, \ldots, n$, are uniformly distributed $U[0,1]$ random variables. Therefore, without loss of generality we can assume that the $X_{i}$ 's and $Y_{i}$ 's are uniformly distributed on $[0,1]$. Then model (2) becomes

$$
H_{\theta}(x, y)=x y+\theta \Omega(x, y), \quad(x, y) \in I^{2}
$$


Model (2) is due to Farlie [3]. For $\theta>0(\theta<0)$, it is an important particular case of the alternative of positive (negative) quadrant dependence. Recall that $H$ is said to be positively (negatively) quadrant dependent if

$$
H(x, y) \geq(\leq) F(x) G(y) \quad \text { for all }(x, y) \in \mathbb{R}^{2} .
$$

The above definition of positive (negative) quadrant dependence is equivalent to requiring for all $(x, y) \in \mathbb{R}^{2}$,

$$
\mathbf{P}(X>x, Y>y) \geq(\leq) \mathbf{P}(X>x) \mathbf{P}(Y>y)
$$

That is, the components of $(X, Y)$ are more (less) likely than those of the vector of independent variables having the same univariate distributions as $X$ and $Y$, to take on small and large values simultaneously. Because of the similar character of the testing problems $H_{0}$ versus $H_{1}$ and $H_{0}$ versus $H_{1}^{\prime}$, we deal with the first problem only.

Model (2) is also closely related to some other alternatives to independence widely studied in the literature, see [14]. For example, the famous model of dependence studied by Hájek and Šidák [6] is reduced in essence to model (2) with dependence function

$$
\Omega(x, y)=y f\left(F^{-1}(x)\right)+x g\left(G^{-1}(y)\right)
$$

Indeed, Hájek and Šidák assumed the following alternative to independence:

$$
H_{2}: X_{i}=X_{i}^{*}+\theta Z_{i}, \quad Y_{i}=Y_{i}^{*}+\theta Z_{i}, \quad i=1, \ldots, n
$$

where all the variables $X_{i}^{*}, Y_{i}^{*}, Z_{i}$ are mutually independent and the distribution types of $X_{i}^{*}, Y_{i}^{*}$ are known, the distribution of $Z_{i}$ is arbitrary, their distributions do not depend on $i$, and $\theta$ is a real non-negative parameter. To see the correspondence between these two models, assume without loss of generality that $X^{*}$ has a distribution with d.f. $F$ and p.d.f. $f, Y^{*}$ has a distribution with d.f $G$ and p.d.f. $g$, and $Z$ has a uniform $U(0,1)$ distribution. By independence, the joint p.d.f. of $X, Y$, and $Z$ has the form

$$
\begin{aligned}
f_{X, Y, Z}(x, y, z ; \theta) & =f_{X^{*}, Y^{*}, Z}(x-\theta z, y-\theta z, z) \\
& =f(x-\theta z) g(y-\theta z) \mathbb{I}_{(0,1)}(z),
\end{aligned}
$$


and hence the joint p.d.f. of $X$ and $Y$ is equal to

$$
h_{\theta}(x, y)=\int_{\mathbb{R}} f_{X, Y, Z}(x, y, z ; \theta) d z=\int_{0}^{1} f(x-\theta z) g(y-\theta z) d z
$$

Under suitable smoothness conditions on $f$ and $g$, applying the Taylor formula to $f(x-\theta z)$ and $g(y-\theta z)$ in $(6)$, we get the following expression for the corresponding distribution function

$$
\begin{aligned}
H_{\theta}(x, y) & =\int_{-\infty}^{x} \int_{-\infty}^{y} h_{\theta}(u, v) d u d v \\
& =F(x) G(y)-\frac{\theta}{2}(F(x) g(y)+f(x) G(y))+O\left(\theta^{2}\right) \\
& =F(x) G(y)-\frac{\theta}{2} \Omega(F(x), G(y))+O\left(\theta^{2}\right) .
\end{aligned}
$$

with $\Omega$ given by (4).

Similar, but more general alternative was studied in [19]. It has the form

$$
H_{3}: X_{i}=X_{i}^{*}+\theta q\left(X_{i}^{*}\right) Z_{i}, \quad Y_{i}=Y_{i}^{*}+\theta Z_{i}
$$

with an arbitrary monotonic function $q$. The most important is the case of an indicator function $q$; in this case, test statistics are computed from an incomplete data. Using the above arguments, it is easy to show that the alternative $H_{3}$ essentially corresponds to our model with

$$
\Omega(x, y)= \begin{cases}y f\left(F^{-1}(x)\right) / q\left(F^{-1}(x)\right)+x g\left(G^{-1}(y)\right) & , q(x) \neq 0 \\ y f\left(F^{-1}(x)\right) & , q(x)=0 .\end{cases}
$$

Another well-known model to independence is the Tukey-Huber model. It has the form

$$
H_{3}: H_{\theta}(x, y)=(1-\theta) F(x) G(y)+\theta \tilde{H}(x, y), \quad(x, y) \in \mathbb{R}^{2}
$$

where $F(x)$ and $G(y)$ are fixed d.f., $\tilde{H}(x, y)$ is an arbitrary d.f., and $\theta \in(0,1)$. With

$$
\Omega(x, y)=\tilde{H}(x, y)-F(x) G(y)
$$

model (2) becomes (7). Thus, the Tukey-Huber model is a particular case of model (2) studied in this thesis. 
To make the function $H_{\theta}(x, y)$ in (3) a distribution function, we need some restrictions on $\Omega(x, y)$. Below we require that $\Omega$ vanishes on the boundary of the cube $I^{2}$. This guarantees a proper behaviour of $H_{\theta}(x, y)$ at 0 and 1 . Moreover, the application of general results of the Pitman efficiency theory requires certain regularity conditions: twice continuously differentiable with respect to all variables.

Let $\mathbf{C}_{0}^{2}\left(I^{2}\right)$ be the space of all functions $f(x, y)$ defined on the unit cube $I^{2}=[0,1] \times$ $[0,1]$, twice continuously differentiable with respect to all variables and subject to condition $\left.f(x, y)\right|_{\partial I^{2}}=0$, where $\partial I^{2}$ is the boundary of $I^{2}$, with natural norm

$$
\|f\|=\sup _{x, y}\left|f_{x x y y}\right|+\sum_{|\alpha| \leq 2} \sup \left|D^{\alpha} f\right|
$$

where $\alpha=\left(\alpha_{1}, \alpha_{2}\right),|\alpha|=\alpha_{1}+\alpha_{2}, D^{\alpha} f=\frac{\partial^{|\alpha|} f}{\partial x^{\alpha_{1}} \partial y^{\alpha_{2}}}$. We assume that

(C1) $\Omega(x, y) \geq 0, \quad(x, y) \in I^{2}$;

(C2) $\Omega(x, y) \in \mathbf{C}_{0}^{2}\left(I^{2}\right)$.

Then the density $h_{\theta}(x, y)=1+\theta \omega(x, y)$ corresponding to $H_{\theta}(x, y)$ satisfies

$$
h_{\theta}(x, y)=1+\theta \omega(x, y) \geq 0, \quad \text { for all small } \theta>0
$$

where

$$
\omega(x, y)=\frac{\partial^{2} \Omega(x, y)}{\partial x \partial y}, \quad(x, y) \in I^{2}
$$

Remark. Regularity condition (C2) used for deriving the asymptotic power functions of statistics at hand can be relaxed. More precisely, one can replace it by the following two conditions:

(a) $\left.\Omega(x, y)\right|_{\partial I^{2}}=0$

(b) there exists a non-zero mixed derivative

$$
\omega(x, y)=\frac{\partial^{2} \Omega(x, y)}{\partial x \partial y}=\frac{\partial^{2} \Omega(x, y)}{\partial y \partial x}, \quad \text { for } \lambda \text {-almost all }(x, y) \in I^{2},
$$

such that $\omega \in \mathbf{L}_{2}\left(I^{2}\right)$ and

$$
h_{\theta}(x, y)=1+\theta \omega(x, y) \geq 0, \quad \text { for all small } \theta \geq 0
$$


Here $\mathbf{L}_{2}(A)$ denotes the space of square integrable functions with respect to the Lebesgue measure $\lambda$ on a set $A \in \mathbb{R}^{2}$.

Define $\mathcal{H}=\left\{H_{\theta}\right\}$ to be the class of continuous d.f.'s of the type (3) that satisfy conditions (C1)-(C2). In the sequel, all bivariate d.f's in the thesis assumed to be from $\mathcal{H}$. We denote by $\mathbf{P}_{\theta}$ the distribution with d.f. $H_{\theta}$. The corresponding expectation and the variance are denoted $\mathbf{E}_{\theta}$ and $\operatorname{Var}_{\theta}$. We use $\mathbb{I}_{A}$ for the indicator of a set $A$. The symbol $N\left(\mu, \sigma^{2}\right)$ is reserved for the normal distribution with mean $\mu$ and variance $\sigma^{2}$. The standard normal density and the corresponding distribution function are denoted by $\varphi$ and $\Phi$, respectively. The expression $o_{\mathbf{P}}(1)$ denotes a sequence of random variables that convergence to zero in P-probability. Below $c, C$ with and without indices are used for various constants.

For testing independence, we use Blest's correlation coefficient $\nu_{n}$ and its symmetrized variant $s_{n}$. Our aim is to find the Pitman efficiency of the corresponding tests for a general class of alternatives given by (2) and compare these statistics with the other analogous measures of association existing in the literature, including the so-called "top-down" coefficient of Iman and Conover [9] and the weighted Kendall's and Spearman's statistics [19], [14]. Moreover, we will establish conditions under which the Blest type tests are asymptotically optimal. 


\section{Chapter 3}

\section{Rank Statistics for Independence}

A rank statistic is a (measurable) function of ranks. Rank statistics are particularly useful in various problems of testing hypotheses since the corresponding tests are distribution free and suffer negligible efficiency loss in comparison with their parametric counterparts; also, such tests may be much more efficient in heavy-tailed models. In this section, we present some basic results on the limiting behavior of linear rank statistics for independence.

\subsection{Linear Rank Statistics}

Let $X_{1}, \ldots, X_{n}$ be a random sample from a continuous distribution with d.f. $F(x)$. Let us arrange $X_{1}, \ldots, X_{n}$ in the order from the smallest to the largest

$$
X_{(1)}<X_{(2)}<\ldots<X_{(n)}
$$

thus getting the set of order statistics. (Here, we use strict inequalities, because being drawn from a continuous distribution, $X_{1}, \ldots, X_{n}$ assume different values with probability 1.) The statistic $X_{(i)}$ is called the $i$ th order statistic; and the position of $X_{i}$ in the set (8), $R_{i}$, is called the rank of $X_{i}$.

Now, let $\left(X_{1}, Y_{1}\right), \ldots,\left(X_{n}, Y_{n}\right)$ be independent identically distributed bivariate vectors, with continuous d.f. $H$ and marginal d.f.'s $F$ and $G$. Suppose it is desired to determine whether, within each pair, $X_{i}$ and $Y_{i}$ are independent. To test the hypothesis of independence, $H_{0}: H \equiv F G$, linear rank statistics are often used. They are defined as follows. 
Denote by $R_{i}$ the rank of $X_{i}$ among $X_{1}, \ldots, X_{n}$ and by $Q_{i}$ the rank of $Y_{i}$ among $Y_{1}, \ldots, Y_{n}$. A linear rank statistic is a statistic of the form

$$
T_{n}=\frac{1}{n} \sum_{i=1}^{n} a_{n}\left(R_{i}, Q_{i}\right),
$$

where $a_{n}(i, j)$ are the numbers called scores. The symmetrized version of Blest's correlation coefficient $s_{n}$ is of this type. Let $F_{n}, G_{n}$ and $H_{n}$ be empirical d.f.'s defined by

$$
\begin{aligned}
F_{n}(x) & =\frac{1}{n} \sum_{i=1}^{n} \mathbb{I}\left\{X_{i} \leq x\right\}, \quad G_{n}(y)=\frac{1}{n} \sum_{i=1}^{n} \mathbb{I}\left\{Y_{i} \leq x\right\} \\
H_{n}(x, y) & =\frac{1}{n} \sum_{i=1}^{n} \mathbb{I}\left\{X_{i} \leq x, Y_{i} \leq y\right\} .
\end{aligned}
$$

An alternative expression for $T_{n}$ is given by [16]

$$
T_{n}=\iint_{\mathbb{R}^{2}} L_{n}\left(F_{n}(x), G_{n}(y)\right) d H_{n}(x, y)
$$

where $L_{n}$ is defined (and finite) on $(0,1] \times(0,1]$ by

$$
L_{n}(x, y)=a_{n}(i, j), \quad(x, y) \in((i-1) / n, i / n] \times((j-1) / n, j / n], \quad i, j=1, \ldots, n
$$

This can be easily seen from the identities

$$
F_{n}\left(X_{i}\right)=\frac{R_{i}}{n}, \quad G_{n}\left(Y_{i}\right)=\frac{Q_{i}}{n}, \quad H_{n}\left(X_{i}, Y_{i}\right)=\frac{1}{n} \sum_{j=1}^{n} \mathbb{I}\left\{R_{j} \leq R_{i}, Q_{j} \leq Q_{i}\right\},
$$

and the representation

$$
\iint_{\mathbb{R}^{2}} \xi(x, y) d H_{n}(x, y)=\frac{1}{n} \sum_{i=1}^{n} \xi\left(X_{i}, Y_{i}\right)
$$

The function $L_{n}$ is called the score function. If for some function $L(x, y)$ defined (and finite) on $(0,1) \times(0,1)$,

$$
\begin{gathered}
L_{n}(x, y)=L(i /(n+1), j /(n+1)) \\
(x, y) \in((i-1) / n, i / n] \times((j-1) / n, j / n], \quad i, j=1, \ldots, n,
\end{gathered}
$$

then $L_{n}$ is called an approximate score function derived from $L$ (for the sample size $n$ ). 
Many well-known statistics for testing independence, such as Spearman's $\rho$, are of more simple form

$$
\left.V_{n}=\frac{1}{n} \sum^{n^{-}} b_{n}^{n}\left(R_{i}\right) c_{n}\left(Q_{i}\right), Q_{i}\right),
$$

where $b_{n}(i)$ and $c_{n}(i), i=1, \ldots, n$, are real numbers. Blest's correlation coefficient $\nu_{n}$ is also of this type. Similarly, we can show that an alternative expression for $V_{n}$ is

$$
V_{n}=\iint_{\mathbb{R}^{2}} J_{n}\left(F_{n}(x)\right) K_{n}\left(G_{n}(y)\right) d H_{n}(x, y)
$$

where

$$
J_{n}(x)=b_{n}(i), \quad K_{n}(x)=c_{n}(i), \quad(i-1) / n<x \leq i / n, \quad i=1, \ldots, n
$$

In practice, one often takes

$$
J_{n}(x)=J(i /(n+1)), \quad K_{n}(x)=K(i /(n+1)), \quad(i-1) / n<x \leq i / n,
$$

with some score generating functions $J(x)$ and $K(x)$.

\subsection{Asymptotic Normality of Linear Rank Statistics}

Asymptotic theory of rank statistics for independence was essentially developed by Hájek and Šidák in the 1960's [6], and then extended by Ruymgaart, Shorack and van Zwet in the 1970's, see [15], [16]. It was shown that, in case of sampling from a continuous bivariate distribution, under certain restrictions on the limiting score functions $L, J$, and $K$, properly normalized statistics $T_{n}$ and $V_{n}$ are asymptotically normal. The rate of growth of $L$ near the boundary of the unit cube $[0,1] \times[0,1]$ and the rate of growth of $J$ and $K$ near 0 and 1 play an important role among the conditions for asymptotic normality of the standardized $T_{n}$ and $V_{n}$. Besides this growth, some smoothness condition of the functions $L, J$, and $K$ are needed. In particular, if $J$ and $K$ are continuously differentiable on $(0,1)$, and the derivatives satisfy

$$
\left|J^{(i)}(x)\right| \leq C r^{\alpha+i}(x), \quad\left|K^{(i)}(x)\right| \leq C r^{\beta+i}(x), \quad i=0,1, \quad x \in(0,1),
$$


where $C$ is a positive constant, $r(x)=1 /[x(1-x)]$, and $\alpha$ and $\beta$ satisfy

$$
\alpha=(1 / 2-\delta) / p_{0}, \quad \beta=(1 / 2-\delta) / q_{0},
$$

for some $0<\delta<1 / 2$ and some $p_{0}, q_{0}>1$ with $1 / p_{0}+1 / q_{0}=1$, then, see [15], Theorem 2.1 ,

$$
\sqrt{n}\left(V_{n}-\mu_{V}\right) \stackrel{\mathrm{d}}{\rightarrow} N\left(0, \sigma_{V}^{2}\right), \quad n \rightarrow \infty
$$

where

$$
\begin{aligned}
\mu_{V} & =\mathbf{E}\{J(F(X)) K(G(Y))\} \\
\sigma_{V}^{2} & =\operatorname{Var}\{J(F(X)) K(G(Y)) \\
& +\iint_{\mathbb{R}^{2}}(\mathbb{I}\{F(x) \geq F(X)\}-F(x)) J^{\prime}(F(x)) K(G(y)) d H(x, y) \\
& \left.+\iint_{\mathbb{R}^{2}}(\mathbb{I}\{G(y) \geq G(Y)\}-G(y)) J(F(x)) K^{\prime}(G(y)) d H(x, y)\right\}
\end{aligned}
$$

An extension of this result to the case of statistic $T_{n}$, was obtained by Ruymgaart, see [16], Theorem 2.1.1. Under similar smoothness conditions and assumptions on the growth of $L$ and its first-order derivatives $L_{x}$ and $L_{y}$,

$$
\sqrt{n}\left(T_{n}-\mu_{T}\right) \stackrel{\mathrm{d}}{\rightarrow} N\left(0, \sigma_{T}^{2}\right), \quad n \rightarrow \infty
$$

where

$$
\begin{aligned}
\mu_{T} & =\mathbf{E}\{L(F(X), G(Y))\} \\
\sigma_{T}^{2} & =\operatorname{Var}\{L(F(X), G(Y)) \\
& +\iint_{\mathbb{R}^{2}}(\mathbb{I}\{F(x) \geq F(X)\}-F(x)) L_{x}(F(x), G(y)) d H(x, y) \\
& \left.+\iint_{\mathbb{R}^{2}}(\mathbb{I}\{G(y) \geq G(Y)\}-G(y)) L_{y}(F(x), G(y)) d H(x, y)\right\} .
\end{aligned}
$$

The results presented in this section will be used for deriving the limiting normality of the tests under consideration. 


\section{Chapter 4}

\section{Blest's Measure of Rank Correlation}

There are numerous situations in which $n$ objects are ranked by two or more independent sources, where interest centers primarily on agreement in the top rankings, and disagreements at the bottom of the rankings are of little or no importance. Statistics such as Spearman's $\rho$ and Kendall's $\tau$ do not provide an appropriate measure of agreement in such situations, because they give all ranks equal weighting. In this section, we introduce Blest's measure of rank correlation, which is sensitive to discrepancies among the first ranks.

\subsection{Blest's Measure of Rank Correlation}

Blest's measure of rank correlation was developed from a graphical approach by Blest [1]. It attaches more significance to early ranking of an initially given order. Next, we illustrate how it was developed.

Assume that $R_{i}=i, i=1, \ldots, n$, and consider accumulated ranks across two rankings and, in particular, the differences between the two sets; that is,

$$
v_{k}=\sum_{i=1}^{k} Q_{i}-\sum_{i=1}^{k} i, \quad k=1, \ldots, n .
$$

The total of these differences between the two accumulated ranks,

$$
V=\sum_{k=1}^{n} v_{k}=\sum_{k=1}^{n} \sum_{i=1}^{k}\left(Q_{i}-i\right),
$$

offers a measure of the disarray of originally ordered data. If $Q_{i}=i$, for all $i=1, \ldots, n$, then $V$ attains its minimum value $V_{\min }=0$. The maximum value for $V$, obtained for inverse 
Table 2: The accumulated ranks and their differences, for $n=6$ and $k=1, \ldots, 6$

\begin{tabular}{|c|c|c|c|c|c|c|}
\hline \hline$i$ & 1 & 2 & 3 & 4 & 5 & 6 \\
\hline$\sum_{i=1}^{k} i$ & 1 & 3 & 6 & 10 & 15 & 21 \\
\hline$Q_{i}$ & 2 & 3 & 1 & 5 & 6 & 4 \\
\hline$\sum_{i=1}^{k} Q_{i}$ & 2 & 5 & 6 & 11 & 17 & 21 \\
\hline$\sum_{i=1}^{k} Q_{i}-\sum_{i=1}^{k} i$ & 1 & 2 & 0 & 1 & 2 & 0 \\
\hline$\sum_{i=1}^{k}(n+1-i)-\sum_{i=1}^{k} i$ & 5 & 8 & 9 & 8 & 5 & 0 \\
\hline
\end{tabular}

natural order when $Q_{i}=n+1-i$ is

$$
V_{\max }=\sum_{k=1}^{n} \sum_{i=1}^{k}((n+1-i)-i)=\frac{n\left(n^{2}-1\right)}{6} .
$$

For example, for $n=6$, we consider two rankings, the natural order $1,2,3,4,5,6$ and its permutation set $2,3,1,5,6,4$. The two sets of accumulated ranks are 1,3,6,10,15, 21 and $2,5,6,11,17,21$, respectively. The differences between ranks are, successively, $1,2,0,1,2,0$ with total $V=6$. For the inverse natural order the accumulated differences are $5,8,9,8,5,0$ with $V_{\max }=35$. Table 2 summarizes the two sets of accumulated ranks and their differences. We plot the deviations in cumulative rank for a random permutation against the cumulative rank for natural order. The polygons in Figure 1 show the areas representing deviation from natural order.

It can be checked that the above area calculations give greater areas and hence greater weight to transpositions for larger values of $k$ in $1, \ldots, n$. For example, a single transposition on the natural order for $n=6$ yielding $2,1,3,4,5,6$ gives an area 1.5 whereas $1,2,3,4,6,5$ gives 5.5. In order to place emphasis on the relative importance of early rankings, Blest proposed to measure, as an area, the total deviation of cumulative ranks against ranks accumulated in inverse natural order. Under this proposal the polygon, for which the area is a measure of the deviation from natural order, is described by the set of points $(0,0) ;\left[\left(\sum_{i=1}^{k}(n+1-i), \sum_{i=1}^{k} v_{i}, k=1, \ldots, n\right]\right.$. 


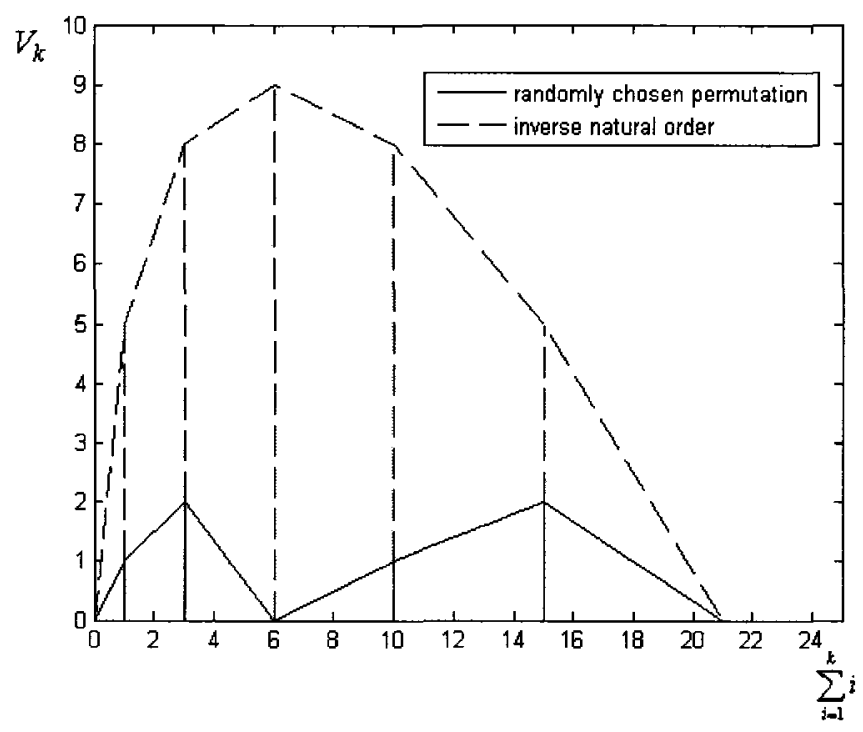

Figure 1: Polygonal areas determined by cumulative ranks for $n=6$ and $k=1, \ldots, 6$

In the general case, the area $W$, under the polygon is given by [1]

$$
W=\frac{1}{2} \sum_{i=1}^{n}(n+1-i)^{2} Q_{i}-\frac{n(n+1)^{2}(n+2)}{24} .
$$

For natural order, $W_{\min }=0$. For inverse natural order,

$$
W_{\max }=\frac{n(n+1)^{2}(n-1)}{12} .
$$

Comparing areas as measures of the disarray of a given permutation, Blest has proposed a new correlation coefficient

$$
\nu_{n}=1-\frac{2 W}{W_{\max }}=1-\frac{24 W}{n(n+1)^{2}(n-1)} .
$$

Using (13) and replacing $i$ by $R_{i}$, one can express Blest's correlation coefficient as follows

$$
\nu_{n}=\frac{2 n+1}{n-1}-\frac{12}{n^{2}-n} \sum_{i=1}^{n}\left(1-\frac{R_{i}}{n+1}\right)^{2} Q_{i} .
$$

Numerical results and calculations reported by Blest [1] show that Blest's statistic can discriminate more easily between individual permutations than either $\rho_{n}$ or $\tau_{n}$ while being highly correlated with both of them. 


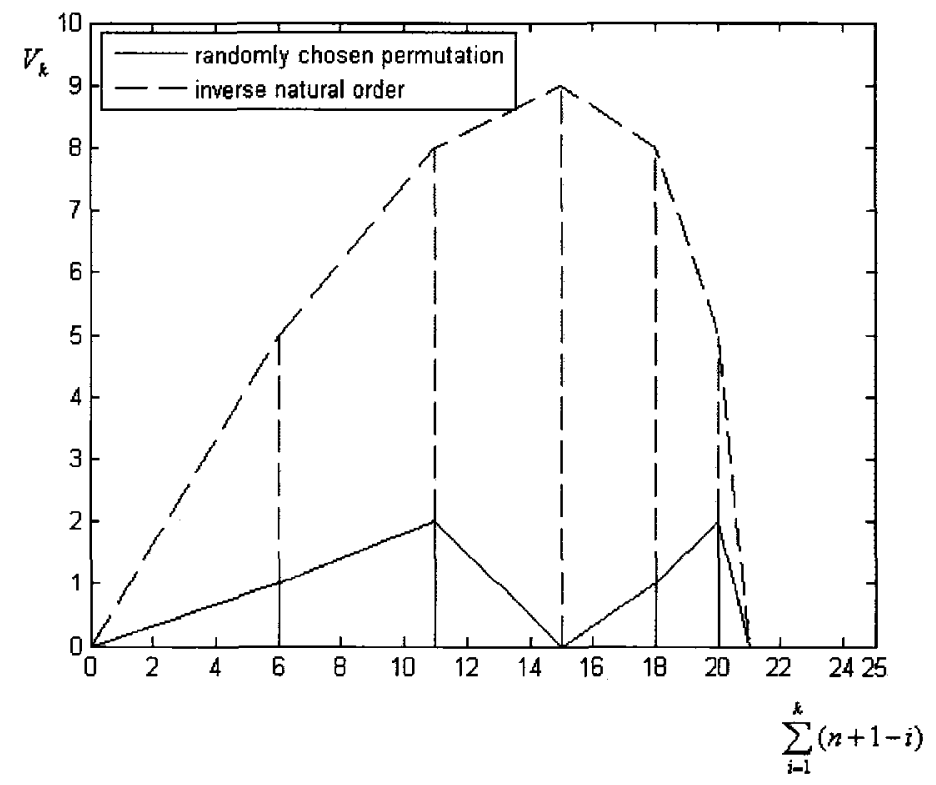

Figure 2: Polygonal areas, revised definition, for $n=6$ and $k=1, \ldots, 6$

\subsection{Symmetrized Version of Blest's Statistics}

There are reasonable conditions for a nonparametric measure of dependence for two continuously distributed random variables $X$ and $Y$. According to Scarsini [17], any concordance measure $\kappa$ should satisfy the following properties:

1. $\kappa$ is defined for every pair $(X, Y)$ of continuous random variables.

2. $-1 \leq \kappa(X, Y) \leq 1, \kappa(X, X)=1$, and $\kappa(X,-X)=-1$.

3. $\kappa(X, Y)=\kappa(Y, X)$.

4. If $X$ and $Y$ are independent, then $\mathbf{E} \kappa(X, Y)=0$.

5. $\kappa(-X, Y)=\kappa(X,-Y)=-\kappa(X, Y)$.

6. If $(X, Y) \prec\left(X^{*}, Y^{*}\right)$ in the positive quadrant dependence ordering, i.e.,

$$
\mathbf{P}(X<x, Y<y) \leq \mathbf{P}\left(X^{*}<x, Y^{*}<y\right), \quad \text { for all }(x, y) \in \mathbb{R}^{2},
$$

then $\kappa(X, Y) \leq \kappa\left(X^{*}, Y^{*}\right)$ 
7. If $\left(X_{1}, Y_{1}\right),\left(X_{2}, Y_{2}\right), \ldots$ is a sequence of continuous random vectors that converges weakly to a pair $(X, Y)$, then $\kappa\left(X_{n}, Y_{n}\right) \stackrel{\mathrm{d}}{\rightarrow} \kappa(X, Y)$, as $n \rightarrow \infty$.

It is well known that both Spearman's $\rho$ and Kendall's $\tau$ satisfy all these conditions. It was shown by Genest \& Plante [5] that the Blest index $\nu$ only has properties $1,2,4,6$ and 7. In general, $\nu(X, Y) \neq \nu(Y, X), \nu(-X, Y) \neq-\nu(X, Y)$, and $\nu(X,-Y) \neq-\nu(X, Y)$. To eliminate the dissymmetry, Genest and Plante [5] proposed a symmetrized version of Blest's coefficient. For this, by analogy with $\nu_{n}$, they defined the statistic

$$
\tilde{\nu}_{n}=\frac{2 n+1}{n-1}-\frac{12}{n^{2}-n} \sum_{i=1}^{n}\left(1-\frac{Q_{i}}{n+1}\right)^{2} R_{i}
$$

and put

$$
s_{n}=\frac{\nu+\tilde{\nu}}{2}=-\frac{4 n+5}{n-1}+\frac{6}{n^{3}-n} \sum_{i=1}^{n} R_{i} Q_{i}\left(4-\frac{R_{i}+Q_{i}}{n+1}\right) .
$$

Genest and Plante [5] showed that the symmetric variant of $\nu_{n}, s_{n}$, which retains the character of Blest's coefficient that greater emphasis should be given to discrepancies in the small ranks, is highly correlated with Spearman's $\rho$ and Kendall's $\tau$. They have also done a study of asymptotic and finite-sample efficiency properties of $s_{n}$ as a test statistic for testing the hypothesis of independence,

$$
H_{0}: H(x, y)=F(x) G(y), \quad(x, y) \in \mathbb{R}^{2},
$$

for several distribution models. In the next section, we calculate asymptotic efficiency of the tests based on $\nu_{n}, \tilde{\nu_{n}}$, and $s_{n}$ for a wide class of alternatives close to the null hypothesis of independence. We also show that for large sample sizes the symmetrized Blest statistic $s_{n}$ often has a better performance than the original one. 


\section{Chapter 5}

\section{Asymptotic Relative Efficiency of the Blest Type}

\section{Tests}

\subsection{Concept of Asymptotic Relative Efficiency}

Sequences of tests can be ranked in quality by using the concept of relative efficiency. Let $X_{1}, \ldots, X_{n}$ be a random sample from a distribution indexed by an unknown parameter $\theta \in \Theta \subseteq \mathbb{R}$. Consider the problem of testing the hypotheses

$$
\begin{aligned}
& H: \theta=\theta_{0} \\
& A: \theta \neq \theta_{0} .
\end{aligned}
$$

Let $N_{T}(\alpha, \beta, \theta)$ be the minimal sample size necessary for the test of level $\alpha$ based on $\left\{T_{n}\right\}$ to have the power not less than $\beta$ at point $\theta \neq \theta_{0}$. Suppose that for testing the null hypothesis $H$ versus the alternative $A$ two sequences of test statistics $\left\{T_{n}\right\}$ and $\left\{V_{n}\right\}$ are available. The relative efficiency of $\left\{V_{n}\right\}$ with respect to $\left\{T_{n}\right\}$ is defined by

$$
e_{V, T}(\alpha, \beta, \theta)=\frac{N_{T}(\alpha, \beta, \theta)}{N_{V}(\alpha, \beta, \theta)}
$$

A value of the relative efficiency $e_{V, T}(\alpha, \beta, \theta)$ larger than 1 indicates that fewer observations for reaching the power $\beta$ at the level $\alpha$ and the alternative value $\theta$ are needed with the sequence $\left\{V_{n}\right\}$, which may then be considered the better one.

Relative efficiency has indisputable merits. Unfortunately, it also has a substantial drawback. The value of $e_{V, T}(\alpha, \beta, \theta)$ depends on three arguments (and two sequences of 
statistics). Therefore it is extremely difficult or simply impossible to calculate this value, except for trivial cases. One can overcome this difficulty by calculating the limiting values of $e_{V, T}(\alpha, \beta, \theta)$ as $\alpha \rightarrow 0$, as $\beta \rightarrow 1$, and as $\theta \rightarrow \theta_{0}$, keeping fixed the values of the two remaining parameters. As a result one obtains three fundamental types of the asymptotic relative efficiency (ARE), see, for example, [12], [21], [18].

1. If for $\beta \in(0,1)$ and $\theta \neq \theta_{0}$ there exists a limit

$$
e_{V, T}^{B}(\beta, \theta):=\lim _{\alpha \downarrow 0} e_{V, T}(\alpha, \beta, \theta)
$$

then this limit is called the Bahadur $A R E$ of the sequence $\left\{V_{n}\right\}$ with respect to $\left\{T_{n}\right\}$.

2. If for $\alpha \in(0,1)$ and $\theta \neq \theta_{0}$ there exists a limit

$$
e_{V, T}^{H L}(\alpha, \theta):=\lim _{\beta \uparrow 1} e_{V, T}(\alpha, \beta, \theta)
$$

then this limit is called the Hodges-Lehmann $A R E$ of the sequence $\left\{V_{n}\right\}$ with respect to $\left\{T_{n}\right\}$.

3. If for $0<\alpha<\beta<1$ and $\theta \rightarrow \theta_{0}$ there exists a limit

$$
e_{V, T}^{P}\left(\alpha, \beta, \theta_{0}\right):=\lim _{\theta \rightarrow \theta_{0}} e_{V, T}(\alpha, \beta, \theta)
$$

then this limit is called the Pitman $A R E$ of the sequence $\left\{V_{n}\right\}$ with respect to $\left\{T_{n}\right\}$.

The Pitman efficiency is a classical and widely used tool in asymptotic comparison of tests. In the 1940's Pitman provided with the regularity conditions that make the calculation of the Pitman efficiency quite easy in many cases. In order to calculate the Pitman efficiency $e_{V, T}^{P}\left(\alpha, \beta, \theta_{0}\right)$, one essentially needs the asymptotic normality of the test statistics $T_{n}$ and $V_{n}$ under the null hypothesis and the alternative. More precisely, suppose that $V_{n}$ is a statistic for testing $H_{0}: \theta=0$ versus $H_{1}: \theta>0$ with critical region $\left\{V_{n} \geq k_{n}\right\}$. We will assume the following regularity condition.

\section{Assumptions (Regularity Conditions).}


(R1) There exist functions $\mu$ and $\sigma$ such that for all $z \in \mathbb{R}$ and $\theta_{n}=\delta n^{-1 / 2}, \delta \geq 0$,

$$
\lim _{n \rightarrow \infty} \mathbf{P}_{\theta_{n}}\left(\frac{\sqrt{n}\left(V_{n}-\mu\left(\theta_{n}\right)\right)}{\sigma\left(\theta_{n}\right)}<z\right)=\Phi(z) .
$$

(R2) The function $\mu(\theta)$ is differentiable at zero and

$$
\left.\frac{d}{d \theta} \mu(\theta)\right|_{\theta=0}=\mu^{\prime}(0)>0
$$

(R3) For the sequence $\theta_{n}=\delta / \sqrt{n}$, with some $\delta \geq 0$,

$$
\frac{\sigma\left(\theta_{n}\right)}{\sigma(0)} \rightarrow 1, \quad \frac{\mu^{\prime}\left(\theta_{n}\right)}{\mu^{\prime}(0)} \rightarrow 1, \quad n \rightarrow \infty .
$$

Under conditions (R1)-(R3), the quantity

$$
c=\lim _{n \rightarrow \infty} \frac{\mu^{\prime}\left(\theta_{n}\right)}{\sigma\left(\theta_{n}\right)}=\frac{\mu^{\prime}(0)}{\sigma(0)}
$$

is called the slope or efficacy of the test based on $V_{n}$. For large $n$, it measures the rate of change of the "asymptotic" mean of $V_{n}$ at the null hypothesis. A test with relatively large efficacy $c$ is responding rapidly to alternatives and therefore is expected to have good local power properties.

Now, denote the power function of the test based on $V_{n}$ that rejects the null hypothesis for large values of $V_{n}$ by $\pi_{n}(\theta)$ :

$$
\pi_{n}(\theta)=\mathbf{P}_{\theta}\left(V_{n} \geq k_{n}\right)
$$

A sequence of tests based on $\left\{V_{n}\right\}$ for testing $H_{0}: \theta=0$ versus $H_{1}: \theta>0$ is called asymptotically of level $\alpha$ if

$$
\limsup _{n \rightarrow \infty} \pi_{n}(0) \leq \alpha
$$

Assume that $\theta=\theta_{n}=\delta / \sqrt{n}$ and consider the local limiting power function

$$
\pi(\delta)=\lim _{n \rightarrow \infty} \pi_{n}\left(\frac{\delta}{\sqrt{n}}\right), \quad \delta \geq 0 .
$$

The following result is due to Pitman. It yields an expression for $\pi(\delta)$, see, for example, [21]. 
Theorem 1. Let $\left\{V_{n}\right\}$ be a sequence of statistics used for testing $H_{0}: \theta=0$ versus $H_{1}: \theta>0$ that rejects $H_{0}: \theta=0$ for large values of $V_{n}$. Assume that $V_{n}$ satisfies the regularity conditions (R1)-(R3). Then the power functions $\pi_{n}$ of the tests based on $V_{n}$ that are asymptotically of level $\alpha$ satisfy

$$
\lim _{n \rightarrow \infty} \pi_{n}\left(\frac{\delta}{\sqrt{n}}\right)=1-\Phi\left(z_{\alpha}-\delta \frac{\mu^{\prime}(0)}{\sigma(0)}\right), \quad \delta \geq 0,
$$

where $\Phi$ is the d.f. of a standard normal distribution and $z_{\alpha}=\Phi^{-1}(1-\alpha)$.

The next theorem is often used for calculating the asymptotic relative efficiency of two tests, see, for example, [7], [12], [21].

Theorem 2. Let $T_{n, 1}$ and $T_{n, 2}$ provide two tests of $H_{0}: \theta=0$ versus $H_{1}: \theta>0$ that reject $H_{0}: \theta=0$ for large values of $T_{n, 1}$ and $T_{n, 2}$. Suppose they both satisfy the regularity conditions (R1)-(R3). Then the Pitman asymptotic efficiency of $\left\{T_{n, 1}\right\}$ relative to $\left\{T_{n, 2}\right\}$ is

$$
e\left(T_{1}, T_{2}\right)=\frac{c_{1}^{2}}{c_{2}^{2}}=\left(\frac{\mu_{1}^{\prime}(0) / \sigma_{1}(0)}{\mu_{2}^{\prime}(0) / \sigma_{2}(0)}\right)^{2}
$$

In this project, we will use the Pitman approach to calculate the ARE, because, as it will be shown below, our test statistics are asymptotically normal under the null hypothesis and the alternative.

\subsection{Asymptotic Normality of the Blest Type Tests}

In this section, we derive the asymptotic distributions of $\nu_{n}, \tilde{\nu}_{n}$, and $s_{n}$. Note that the asymptotic distribution of $\nu_{n}$ is that of the statistic

$$
\nu_{n}^{*}=2-\frac{12}{n} \sum_{i=1}^{n}\left(1-\frac{R_{i}}{n+1}\right)^{2} \frac{Q_{i}}{n+1}=2-12 V_{n},
$$

where

$$
V_{n}=\frac{1}{n} \sum_{i=1}^{n}\left(1-\frac{R_{i}}{n+1}\right)^{2} \frac{Q_{i}}{n+1}
$$


Put

$$
J(x)=(1-x)^{2}, \quad K(x)=x, \quad 0 \leq x \leq 1,
$$

and define the score functions $J_{n}$ and $K_{n}$ as follows:

$$
J_{n}(x)=J(i /(n+1)) \quad K_{n}(x)=K(i /(n+1)), \quad(i-1) / n<x \leq i / n
$$

Then statistic (14) is a linear rank statistic of the form

$$
V_{n}=\frac{1}{n} \sum_{i=1}^{n} J_{n}\left(R_{i}\right) K_{n}\left(Q_{i}\right)
$$

Due to $(11), V_{n}$ is asymptotically normal under both, the null hypothesis and the alternative:

$$
\sqrt{n}\left(V_{n}-\mu_{V}(\theta)\right) \stackrel{\mathrm{d}}{\rightarrow} N\left(0, \sigma_{V}^{2}(\theta)\right), \quad \theta \geq 0
$$

where

$$
\begin{aligned}
\mu_{V}(\theta) & =\iint_{I^{2}} J(x) K(y) d H_{\theta}(x, y) \\
\sigma_{V}^{2}(\theta) & =\operatorname{Var}_{\theta}\left\{J(X) K(Y)+\iint_{I^{2}}\left(\mathbb{I}_{[X, 1]}(x)-x\right) J^{\prime}(x) K(y) d H_{\theta}(x, y)\right. \\
& \left.+\iint_{I^{2}}\left(\mathbb{I}_{[Y, 1]}(y)-y\right) J(x) K^{\prime}(y) d H_{\theta}(x, y)\right\} .
\end{aligned}
$$

From this, using Slutsky's theorem,

$$
\sqrt{n}\left(\nu_{n}-\left(2-12 \mu_{V}(\theta)\right)\right) \stackrel{\mathrm{d}}{\rightarrow} N\left(0,144 \sigma_{V}^{2}(\theta)\right) .
$$

For the distribution model (3), the quantity $\mu_{V}(\theta)$ is equal to

$$
\begin{aligned}
\mu_{V}(\theta) & =\iint_{I^{2}}(1-x)^{2} y d H_{\theta}(x, y) \\
& =\iint_{I^{2}}(1-x)^{2} y d x d y+\theta \iint_{I^{2}}(1-x)^{2} y \omega(x, y) d x d y \\
& =1 / 6+\theta \iint_{I^{2}}(1-x)^{2} y \omega(x, y) d x d y
\end{aligned}
$$


The variance of $V_{n}$ is equal to

$$
\begin{aligned}
\sigma_{V}^{2}(\theta) & =\operatorname{Var}_{\theta}\left\{(1-X)^{2} Y-2 \iint_{I^{2}}\left(\mathbb{I}_{[X, 1]}(x)-x\right)(1-x) y d H_{\theta}(x, y)\right. \\
& \left.+\iint_{I^{2}}\left(\mathbb{I}_{[Y, 1]}(y)-y\right)(1-x)^{2} d H_{\theta}(x, y)\right\} \\
& =\operatorname{Var}_{\theta}\left\{(1-X)^{2} Y-2 \iint_{I^{2}} \mathbb{I}_{[X, 1]}(x)(1-x) y d x d y\right. \\
& +\iint_{I^{2}} \mathbb{I}_{[Y, 1]}(y)(1-x)^{2} d x d y-2 \theta \iint_{I^{2}} \mathbb{I}_{[X, 1]}(x)(1-x) y \omega(x, y) d x d y \\
& \left.+\theta \iint_{I^{2}} \mathbb{I}_{[Y, 1]}(y)(1-x)^{2} \omega(x, y) d x d y\right\} \\
& =\operatorname{Var}_{\theta}\left\{(1-X)^{2} Y-\frac{1}{2}(1-X)^{2}+\frac{1}{3}(1-Y)\right. \\
& \left.-2 \theta \iint_{[X, 1] \times[0,1]}(1-x) y \omega(x, y) d y d x+\theta \iint_{[Y, 1] \times[0,1]}(1-x)^{2} \omega(x, y) d x d y\right\} \\
& =\mathbf{E}_{\theta}\left\{Q_{1}(X, Y)+\theta Q_{2}(X, Y)\right\}^{2}-\mathbf{E}_{\theta}^{2}\left\{Q_{1}(X, Y)+\theta Q_{2}(X, Y)\right\},
\end{aligned}
$$

where

$$
\begin{aligned}
Q_{1}(X, Y) & =(1-X)^{2} Y-\frac{1}{2}(1-X)^{2}+\frac{1}{3}(1-Y) \\
Q_{2}(X, Y) & =\iint_{[Y, 1] \times[0,1]}(1-x)^{2} \omega(x, y) d x d y-2 \iint_{[X, 1] \times[0,1]}(1-x) y \omega(x, y) d y d x
\end{aligned}
$$

Hence

$$
\sigma_{V}^{2}(\theta)=\sigma_{V}^{2}(0)+C_{1} \theta+C_{2} \theta^{2}+C_{3} \theta^{3}+C_{4} \theta^{4}
$$

where the $C_{i}$ 's are constants and

$$
\begin{aligned}
\sigma_{V}^{2}(0) & =\mathbf{E}_{0} Q_{1}^{2}(X, Y)-\mathbf{E}_{0}^{2} Q_{1}(X, Y) \\
& =\iint_{I^{2}}\left\{(1-x)^{2} y-\frac{(1-x)^{2}}{2}+\frac{(1-y)}{3}\right\}^{2} d x d y \\
& -\left(\iint_{I^{2}}\left\{(1-x)^{2} y-\frac{(1-x)^{2}}{2}+\frac{(1-y)}{3}\right\} d x d y\right)^{2}=\frac{1}{135}
\end{aligned}
$$

Combining (15), (16), and (17) we arrive at the following limiting relation:

$$
\sqrt{n}\left(\nu_{n}-\mu_{\nu}(\theta)\right) \stackrel{\mathrm{d}}{\rightarrow} N\left(0, \sigma_{\nu}^{2}(\theta)\right)
$$


where

$$
\begin{aligned}
\mu_{\nu}(\theta) & =-12 \theta \iint_{I^{2}}(1-x)^{2} y \omega(x, y) d x d y=24 \theta \iint_{I^{2}}(1-x) \Omega(x, y) d x d y \\
\sigma_{\nu}^{2}(\theta) & =16 / 15+C_{1} \theta+C_{2} \theta^{2}+C_{3} \theta^{3}+C_{4} \theta^{4}
\end{aligned}
$$

By the symmetry, the statistic $\tilde{\nu}_{n}$ satisfies

$$
\sqrt{n}\left(\tilde{\nu}_{n}-\mu_{\tilde{\nu}}(\theta)\right) \stackrel{\mathrm{d}}{\rightarrow} N\left(0, \sigma_{\tilde{\nu}}^{2}(\theta)\right)
$$

where

$$
\begin{aligned}
\mu_{\tilde{\nu}}(\theta) & =-12 \theta \iint_{I^{2}} x(1-y)^{2} \omega(x, y) d x d y=24 \theta \iint_{I^{2}}(1-y) \Omega(x, y) d x d y \\
\sigma_{\tilde{\nu}}^{2}(\theta) & =16 / 15+\tilde{C}_{1} \theta+\tilde{C}_{2} \theta^{2}+\tilde{C}_{3} \theta^{3}+\tilde{C}_{4} \theta^{4}
\end{aligned}
$$

Now we turn to the statistic $s_{n}$. The asymptotic distribution of $s_{n}$ is the same as that of

$$
s_{n}^{*}=-4+\frac{6}{n} \sum_{i=1}^{n} \frac{R_{i}}{n+1} \frac{Q_{i}}{n+1}\left(4-\frac{R_{i}+Q_{i}}{n+1}\right)=-4+6 S_{n}
$$

where

$$
S_{n}=\frac{1}{n} \sum_{i=1}^{n} \frac{R_{i}}{n+1} \frac{Q_{i}}{n+1}\left(4-\frac{R_{i}+Q_{i}}{n+1}\right) .
$$

Define

$$
L(x, y)=x y(4-x-y), \quad 0 \leq x \leq 1, \quad 0 \leq y \leq 1,
$$

and let the score function $L_{n}$ be as follows:

$$
L_{n}(x, y)=L(i /(n+1), j /(n+1)), \quad(i-1) / n<x \leq i / n,(j-1) / n<y \leq j / n .
$$

The assumptions under which (12) is valid are trivially satisfied in our case. Thus according to (12), $S_{n}$ is asymptotically normal under both, the null hypothesis and the alternative:

$$
\sqrt{n}\left(S_{n}-\mu_{S}(\theta)\right) \stackrel{\mathrm{d}}{\rightarrow} N\left(0, \sigma_{S}^{2}(\theta)\right), \quad \theta \geq 0
$$


where

$$
\begin{aligned}
\mu_{S}(\theta) & =\iint_{I^{2}} L(x, y) d H_{\theta}(x, y) \\
\sigma_{S}^{2}(\theta) & =\operatorname{Var}_{\theta}\left\{L(X, Y)+\iint_{I^{2}}\left(\mathbb{I}_{[X, 1]}(x)-x\right) L_{x}(x, y) d H_{\theta}(x, y)\right. \\
& \left.+\iint_{I^{2}}\left(\mathbb{I}_{[Y, 1]}(y)-y\right) L_{y}(x, y) d H_{\theta}(x, y)\right\}
\end{aligned}
$$

Here $L_{x}$ and $L_{y}$ are partial derivatives of $L$ with respect to $x$ and $y$. Using Slutsky's theorem,

$$
\sqrt{n}\left(s_{n}-\left(6 \mu_{S}(\theta)-4\right)\right) \stackrel{\mathrm{d}}{\rightarrow} N\left(0,36 \sigma_{S}^{2}(\theta)\right)
$$

where

$$
\begin{aligned}
\mu_{S}(\theta) & =\iint_{I^{2}} x y(4-x-y) d H_{\theta}(x, y) \\
& =\iint_{I^{2}} x y(4-x-y) d x d y+\theta \iint_{I^{2}} x y(4-x-y) \omega(x, y) d x d y \\
& =\frac{2}{3}+\theta \iint_{I^{2}} x y(4-x-y) \omega(x, y) d x d y,
\end{aligned}
$$

and the variance of $S_{n}$ is equal to

$$
\begin{aligned}
\sigma_{S}^{2}(\theta) & =\operatorname{Var}_{\theta}\left\{X Y(4-X-Y)+\iint_{I^{2}}\left(\mathbb{I}_{[X, 1]}(x)-x\right)\left(4 y-2 x y-y^{2}\right) d H_{\theta}(x, y)\right. \\
& \left.+\iint_{I^{2}}\left(\mathbb{I}_{[Y, 1]}(y)-y\right)\left(4 x-x^{2}-2 x y\right) d H_{\theta}(x, y)\right\} \\
& =\operatorname{Var}_{\theta}\left\{X Y(4-X-Y)+\iint_{[X, 1] \times[0,1]}\left(4 y-2 x y-y^{2}\right) d y d x\right. \\
& +\iint_{[Y, 1] \times[0,1]}\left(4 x-x^{2}-2 x y\right) d x d y \\
& +\theta \iint_{[X, 1] \times[0,1]}\left(4 y-2 x y-y^{2}\right) \omega(x, y) d y d x \\
& \left.+\theta \iint_{[Y, 1] \times[0,1]}\left(4 x-x^{2}-2 x y\right) \omega(x, y) d x d y\right\} \\
& =\operatorname{Var}_{\theta}\left\{X Y(4-X-Y)-5 X / 3+X^{2} / 2-5 Y / 3+Y^{2} / 2\right. \\
& +\theta \iint_{[X, 1] \times[0,1]}\left(4 y-2 x y-y^{2}\right) \omega(x, y) d y d x \\
& \left.+\theta \iint_{[Y, 1] \times[0,1]}\left(4 x-x^{2}-2 x y\right) \omega(x, y) d x d y\right\} \\
& =\mathbf{E}_{\theta}\left\{B_{1}(X, Y)+\theta B_{2}(X, Y)\right\}^{2}-\mathbf{E}_{\theta}^{2}\left\{B_{1}(X, Y)+\theta B_{2}(X, Y)\right\}
\end{aligned}
$$


with

$$
\begin{aligned}
B_{1}(X, Y) & =X Y(4-X-Y)-\frac{5}{3} X+\frac{1}{2} X^{2}-\frac{5}{3} Y+\frac{1}{2} Y^{2} \\
B_{2}(X, Y) & =\iint_{[X, 1] \times[0,1]}\left(4 y-2 x y-y^{2}\right) \omega(x, y) d y d x \\
& +\iint_{[Y, 1] \times[0,1]}\left(4 x-x^{2}-2 x y\right) \omega(x, y) d x d y .
\end{aligned}
$$

Thus

$$
\sigma_{S}^{2}(\theta)=\sigma_{S}^{2}(0)+C_{1} \theta+C_{2} \theta^{2}+C_{3} \theta^{3}+C_{4} \theta^{4}
$$

where the $C_{i}$ 's are constants and

$$
\begin{aligned}
\sigma_{S}^{2}(0) & =\mathbf{E}_{0} B_{1}^{2}(X, Y)-\mathbf{E}_{0}^{2} B_{1}^{2}(X, Y) \\
& =\iint_{I^{2}}\left\{x y(4-x-y)-\frac{5}{3} x+\frac{1}{2} x^{2}-\frac{5}{3} y+\frac{1}{2} y^{2}\right\}^{2} d x d y \\
& -\left\{\iint_{I^{2}}\left(x y(4-x-y)-\frac{5}{3} x+\frac{1}{2} x^{2}-\frac{5}{3} y+\frac{1}{2} y^{2}\right) d x d y\right\}^{2} \\
& =\frac{31}{1080} .
\end{aligned}
$$

Combining (21), (22), (23) we arrive at the following limiting relation for the symmetrized statistic $s_{n}$ :

$$
\sqrt{n}\left(s_{n}-\mu_{s}(\theta)\right) \stackrel{\mathrm{d}}{\rightarrow} N\left(0, \sigma_{s}^{2}(\theta)\right)
$$

with

$$
\begin{aligned}
& \mu_{s}(\theta)=6 \theta \iint_{I^{2}} x y(4-x-y) \omega(x, y) d x d y=6 \theta \iint_{I^{2}}(4-2 x-2 y) \Omega(x, y) d x d y \\
& \sigma_{s}^{2}(\theta)=31 / 30+c_{1} \theta+c_{2} \theta^{2}+c_{3} \theta^{3}+c_{4} \theta^{4}
\end{aligned}
$$

The results of this section will be used to prove Lemmas 1-3 below. 


\subsection{Local Limiting Power Functions of the Blest Type Tests}

According to the results of the previous section, each of the functions $\mu_{\nu}(\theta), \mu_{\tilde{\nu}}(\theta)$, and $\mu_{s}(\theta)$ is differentiable at $\theta=0$ and by (18), (19), and (24)

$$
\begin{aligned}
& \mu_{\nu}^{\prime}(0)=-12 \iint_{I^{2}}(1-x)^{2} y \omega(x, y) d x d y \\
& \mu_{\tilde{\nu}}^{\prime}(0)=-12 \iint_{I^{2}} x(1-y)^{2} \omega(x, y) d x d y \\
& \mu_{s}^{\prime}(0)=6 \iint_{I^{2}} x y(4-x-y) \omega(x, y) d x d y
\end{aligned}
$$

Using the integration by parts technique, $\mu_{\nu}^{\prime}(0), \mu_{\tilde{\nu}}^{\prime}(0)$ and $\mu_{s}^{\prime}(0)$ can be written in terms of function $\Omega$,

$$
\begin{aligned}
& \mu_{\nu}^{\prime}(0)=24 \iint_{I^{2}}(1-x) \Omega(x, y) d x d y \\
& \mu_{\tilde{\nu}}^{\prime}(0)=24 \iint_{I^{2}}(1-y) \Omega(x, y) d x d y \\
& \mu_{s}^{\prime}(0)=12 \iint_{I^{2}}(2-x-y) \Omega(x, y) d x d y
\end{aligned}
$$

Also, being the square root of a polynomial of degree four in $\theta$, each of the standard deviations $\sigma_{\nu}(\theta), \sigma_{\tilde{\nu}}(\theta)$, and $\sigma_{s}(\theta)$ is continuous at $\theta=0$ and

$$
\sigma_{\nu}(0)=\sigma_{\tilde{\nu}}(0)=\sqrt{16 / 15} \quad \sigma_{s}(0)=\sqrt{31 / 30}
$$

The derivatives $\mu_{\nu}^{\prime}(0), \mu_{\tilde{\nu}}^{\prime}(0)$ and $\mu_{s}^{\prime}(0)$ are positive. The relations $\frac{\sigma\left(\theta_{n}\right)}{\sigma(0)} \rightarrow 1$ and $\frac{\mu^{\prime}\left(\theta_{n}\right)}{\mu^{\prime}(0)} \rightarrow 1$, as $n \rightarrow \infty$ hold if $\theta=\theta_{n}=\delta n^{-1 / 2}$. Pitman's regularity conditions are satisfied. Thus the Pitman slopes of the tests based on $\nu_{n}, \tilde{\nu}_{n}, s_{n}$ are equal to

$$
\begin{aligned}
& c_{\nu}=\frac{\mu_{\nu}^{\prime}(0)}{\sigma_{\nu}(0)}=-3 \sqrt{15} \iint_{I^{2}}(1-x)^{2} y \omega(x, y) d x d y, \\
& c_{\tilde{\nu}}=\frac{\mu_{\tilde{\nu}}^{\prime}(0)}{\sigma_{\tilde{\nu}}(0)}=-3 \sqrt{15} \iint_{I^{2}} x(1-y)^{2} \omega(x, y) d x d y, \\
& c_{s}=\frac{\mu_{s}^{\prime}(0)}{\sigma_{s}(0)}=6 \sqrt{30 / 31} \iint_{I^{2}} x y(4-x-y) \omega(x, y) d x d y,
\end{aligned}
$$


or, in terms of function $\Omega$,

$$
\begin{aligned}
& c_{\nu}=\frac{\mu_{\nu}^{\prime}(0)}{\sigma_{\nu}(0)}=6 \sqrt{15} \iint_{I^{2}}(1-x) \Omega(x, y) d x d y, \\
& c_{\tilde{\nu}}=\frac{\mu_{\tilde{\nu}}^{\prime}(0)}{\sigma_{\tilde{\nu}}(0)}=6 \sqrt{15} \iint_{I^{2}}(1-y) \Omega(x, y) d x d y, \\
& c_{s}=\frac{\mu_{s}^{\prime}(0)}{\sigma_{s}(0)}=12 \sqrt{30 / 31} \iint_{I^{2}}(2-x-y) \Omega(x, y) d x d y .
\end{aligned}
$$

It is seen from the above formulas that in case of symmetric dependence function $\Omega(x, y)$, the Blest test statistics $\nu_{n}$ and $\tilde{\nu}_{n}$ have the same slope.

Denote $z_{\alpha}$ the $(1-\alpha)$ quantile of a standard normal distribution. Then due to (18) and (19) the critical regions of the approximate level $\alpha$ tests based on $\nu_{n}$ and $\tilde{\nu}_{n}$ are $\left\{\sqrt{n} \nu_{n}>\right.$ $\left.\sqrt{16 / 15} z_{\alpha}\right\}$ and $\left\{\sqrt{n} \tilde{\nu}_{n}>\sqrt{16 / 15} z_{\alpha}\right\}$, respectively. Also, relation (24) leads to the critical region $\left\{\sqrt{n} s_{n}>\sqrt{31 / 30} z_{\alpha}\right\}$ of the approximate level $\alpha$ test based on $s_{n}$.

Now, let $\pi_{\nu, n}(\theta), \pi_{\tilde{\nu}, n}(\theta)$, and $\pi_{s, n}(\theta)$ be the power functions of the tests based on $\nu_{n}$, $\tilde{\nu}_{n}$, and $s_{n}$, respectively, with approximate level $\alpha$. The following results hold.

Lemma 1. The power function of the test based on $\nu_{n}$ satisfies

$$
\pi_{\nu}(\delta):=\lim _{n \rightarrow \infty} \pi_{\nu, n}\left(\frac{\delta}{\sqrt{n}}\right)=1-\Phi\left(z_{\alpha}+\delta c_{\nu}\right)
$$

Lemma 2. The power function of the test based on $\tilde{\nu}_{n}$ satisfies

$$
\pi_{\tilde{\nu}}(\delta):=\lim _{n \rightarrow \infty} \pi_{\tilde{\nu}, n}\left(\frac{\delta}{\sqrt{n}}\right)=1-\Phi\left(z_{\alpha}+\delta c_{\tilde{\nu}}\right) .
$$

Lemma 3. The power function of the test based on $s_{n}$ satisfies

$$
\pi_{s}(\delta):=\lim _{n \rightarrow \infty} \pi_{s, n}\left(\frac{\delta}{\sqrt{n}}\right)=1-\Phi\left(z_{\alpha}-\delta c_{s}\right)
$$

Lemmas 1-3 follow immediately from Theorem 1. 


\subsection{Comparison of Tests on the Basis of ARE}

The Pitman efficiency theory allows us to compare two sequences of tests by just comparing the sizes of their slopes. According to Theorem 2, the Pitman ARE is simply the square of the quotient of two slopes. Now we compare the tests based on the Blest type statistics with those based on the "top-down" coefficient $r_{n}$ and the weighted Spearman's and Kendall's correlation coefficients.

The measure of "top-down" correlation introduced by Iman and Conover [9] is defined as follows:

$$
r_{n}=\frac{1}{n-\phi(1)}\left(\sum_{i=1}^{n} \phi\left(R_{i}\right) \phi\left(Q_{i}\right)-n\right)
$$

where $\phi(i)=\sum_{j=i}^{n}(1 / j)$ are the Savage scores. The statistic $r_{n}$ centers on agreement in the top rankings. The weighted Spearman's and Kendall's correlation coefficients are given by the formulas [19]:

$$
\begin{gathered}
\rho_{n, w}=\sum_{i=1}^{n} w_{i}\left(R_{i}-\frac{n+1}{2}\right)\left(Q_{i}-\frac{n+1}{2}\right), \\
\tau_{n, w}=\frac{2}{\sum_{i, j} w_{i j}-\sum_{i} w_{i i}} \sum_{1 \leq i \leq j \leq n} w_{i j} \operatorname{sign}\left(R_{i}-R_{j}\right) \operatorname{sign}\left(Q_{i}-Q_{j}\right),
\end{gathered}
$$

where $w_{i}$ and $w_{i j}=w_{j i}$ are bounded weight functions mapping the sets $\mathbb{N}$ and $\mathbb{N}^{2}$, respectively, into $\mathbb{R}$. Clearly, when rearranging the initial data sets such that the $X$ values appear in an increasing order we do not change the values of rank correlation coefficients. Therefore, without loss of generality, we can assume that $R_{i}=i, i=1, \ldots, n$. With such an assumption, in various practical applications where interest focuses primarily on agreement in the top rankings, the function $w_{i j}$ has the form $w_{i} w_{j}$, where $w_{i}$ is an indicator. In particular, one often takes

$$
w_{i}=\mathbb{I}\{i \leq[(n+1) p]\}, \quad p \in(0,1] .
$$

The case $p=1$ corresponds to the classical $\tau_{n}$ and $\rho_{n}$ (up to a constant). By choosing $w_{i j}=w_{i} w_{j}$, with $w_{i}$ defined in (28), we make weighted $\rho$ and $\tau$ sensitive to discrepancies among the first $100 p \%$ of ranks, and thus similar in applicability to the Blest statistics. 
Under the alternative at hand, the slopes of such weighted Spearman's and Kendall's test statistics, with we denote by $\rho_{n, p}$ and $\tau_{n, p}$, as well as the slope of $r_{n}$ are given by [14]

$$
\begin{aligned}
\frac{\mu_{\rho_{p}}^{\prime}(0)}{\sigma_{\rho_{p}}(0)} & =\frac{12 \int_{0}^{p}(x-1 / 2) \int_{0}^{1}(y-1 / 2) \omega(x, y) d y d x}{\sqrt{p\left(3(1-p)^{3}+p^{2}\right)}} \\
\frac{\mu_{\tau_{p}}^{\prime}(0)}{\sigma_{\tau_{p}}(0)} & =12 \sqrt{p} \iint_{I^{2}} \Omega(x, y) d x d y \\
\frac{\mu_{r}^{\prime}(0)}{\sigma_{r}(0)} & =\iint_{I^{2}} \frac{\Omega(x, y)}{x y} d x d y
\end{aligned}
$$

Using (26) and (29), we can easily get the relative efficiency of the Blest type tests with respect to their counterparts $r_{n}, \tau_{n, p}$, and $\rho_{n, p}$ in terms of dependence function $\Omega$ and/or its mixed derivative $\omega$. For example,

$$
\begin{aligned}
e(\nu, r) & =540 \frac{\left(\iint_{I^{2}}(1-x) \Omega(x, y) d x d y\right)^{2}}{\left(\iint_{I^{2}} \Omega(x, y) /(x y) d x d y\right)^{2}} \\
e\left(s, \tau_{p}\right) & =\frac{30}{31 p} \frac{\left(\iint_{I^{2}}(2-x-y) \Omega(x, y) d x d y\right)^{2}}{\left(\iint_{I^{2}} \Omega(x, y) d x d y\right)^{2}} \\
e\left(s, \rho_{p}\right) & =\frac{30 p\left(3(1-p)^{3}+p^{2}\right)\left(\iint_{I^{2}}(2-x-y) \Omega(x, y) d x d y\right)^{2}}{31\left(\int_{0}^{p}(x-1 / 2) \int_{0}^{1}(y-1 / 2) \omega(x, y) d y d x\right)^{2}}
\end{aligned}
$$

Example 1. Consider the Farlie-Gumbel-Morgenstern distribution determined by the dependence function

$$
\Omega(x, y)=x(1-x) y(1-y), \quad(x, y) \in I^{2} .
$$

From (25), up to four decimal places,

$$
\left(\mu_{\nu}^{\prime}(0) / \sigma_{\nu}(0)\right)^{2}=\left(\mu_{\tilde{\nu}}^{\prime}(0) / \sigma_{\tilde{\nu}}(0)\right)^{2}=0.1042, \quad\left(\mu_{s}^{\prime}(0) / \sigma_{s}(0)\right)^{2}=0.1075
$$

At the same time, see [13],

$$
\left(\mu_{r}^{\prime}(0) / \sigma_{r}(0)\right)^{2}=0.0625
$$

and

$$
\begin{aligned}
& \left(\mu_{\tau_{p}}^{\prime}(0) / \sigma_{\tau_{p}}(0)\right)^{2}=0.0333,0.0556,0.0778,0.1000,0.1111 \\
& \left(\mu_{\rho_{p}}^{\prime}(0) / \sigma_{\rho_{p}}(0)\right)^{2}=0.0725,0.0889,0.0798,0.0868,0.1111
\end{aligned}
$$


Table 3: Relative efficiencies of the Blest type test statistics versus the top-down test statistic for the Woodworth distribution

\begin{tabular}{c|rrrrrrr}
\hline $\begin{array}{c}\text { Test statistics } \\
\text { compared }\end{array}$ & $k=1$ & $k=2$ & $k=3$ & $k=5$ & $k=7$ & $k=10$ \\
\hline \hline Blest/top-down & 1.66 & 2.33 & 2.73 & 3.16 & 3.37 & 3.53 \\
symmetrized Blest/top-down & 1.72 & 2.40 & 2.82 & 3.26 & 3.48 & 3.64 \\
\hline
\end{tabular}

for $p=0.3,0.5,0.7,0.9,1$, respectively. Therefore,

$$
e(\nu, r)=e(\tilde{\nu}, r) \approx 1.67, \quad e(s, r) \approx 1.72
$$

and

$$
\begin{aligned}
& e\left(s, \tau_{p}\right) \approx 3.23,1.94,1.38,1.08,0.97 \\
& e\left(s, \rho_{p}\right) \approx 1.48,1.21,1.37,1.24,0.97
\end{aligned}
$$

for $p=0.3,0.5,0.7,0.9,1$, respectively. The values obtained demonstrate the superiority of the Blest type tests over the top-down statistic and the weighted $\tau_{p}$ and $\rho_{p}$ in case of sampling from the Farlie-Gumbel-Morgenstern. However, the classical $\tau_{n}$ and $\rho_{n}$ are better than the Blest statistics.

Example 1*. A natural generalization of the Farlie Gumbel-Morgenstern distribution is determined by the dependence function

$$
\Omega_{k}(x, y)=x\left(1-x^{k}\right) y\left(1-y^{k}\right), \quad k=1,2, \ldots, \quad(x, y) \in I^{2} .
$$

This function was first considered by Woodworth [22] and later studied by several authors. It also appeared in [13] in exploring the Bahadur local optimality of the the generalized Kendall's tau introduced by Kochar and Gupta [10].

Tables 3 and 4 contain relative efficiencies of the Blest type tests relative to those based on $r_{n}, \rho_{n, p}$, and $\tau_{n, p}$, for some particular choices of the values of $k$ and $p$. It is seen from the table, that the Blest type tests are not always better than their analogs based on $r_{n}$, $\rho_{n, p}$, and $\tau_{n, p}$. 
Table 4: Relative efficiencies of the symmetrized Blest test statistics versus the weighted Spearman's and Kendall's test statistics for the Woodworth distribution

\begin{tabular}{c|cccccc}
\hline & \multicolumn{6}{|c}{ Efficiency (symmetrized Blest/weighted $\tau$ ) } \\
& $k=1$ & $k=2$ & $k=3$ & $k=5$ & $k=7$ & $k=10$ \\
\hline$p=0.3$ & 3.23 & 2.81 & 2.55 & 2.24 & 2.06 & 1.91 \\
$p=0.5$ & 1.94 & 1.69 & 1.53 & 1.43 & 1.24 & 1.15 \\
$p=0.7$ & 1.38 & 1.20 & 1.09 & 0.96 & 0.88 & 0.82 \\
$p=0.9$ & 1.08 & 0.94 & 0.85 & 0.75 & 0.69 & 0.64 \\
$p=1.0$ & 0.97 & 0.84 & 0.76 & 0.67 & 0.62 & 0.57 \\
\hline \hline & Efficiency (symmetrized Blest/weighted $\rho$ ) \\
& $k=1$ & $k=2$ & $k=3$ & $k=5$ & $k=7$ & $k=10$ \\
\hline$p=0.3$ & 1.48 & 1.86 & 2.18 & 2.62 & 2.85 & 3.03 \\
$p=0.5$ & 1.21 & 1.38 & 1.53 & 1.75 & 1.88 & 1.99 \\
$p=0.7$ & 1.35 & 1.63 & 1.93 & 2.51 & 2.98 & 3.42 \\
$p=0.9$ & 1.24 & 1.29 & 1.40 & 1.75 & 2.26 & 3.37 \\
$p=1.0$ & 0.97 & 0.84 & 0.76 & 0.67 & 0.62 & 0.57 \\
\hline
\end{tabular}


Table 5: Relative efficiencies of the Blest type test statistics versus the top-down test statistic for the normal distribution

\begin{tabular}{c|c}
\hline Test statistics compared & Efficiency \\
\hline \hline Blest/top-down & 1.28 \\
symmetrized Blest/top-down & 1.33 \\
\hline
\end{tabular}

Example 2. Consider the bivariate $N(0,0 ; 1,1 ; \theta)$ standard normal distribution with correlation $\theta$. In this case, the distribution function can be represented by the series, see [2], formula (21.12.6),

$$
H_{\theta}(x, y)=\sum_{k=0}^{\infty} \frac{\Phi^{(k)}(x) \Phi^{(k)}(y)}{k !} \theta^{k}
$$

where $\Phi^{(k)}$ means the $k$ th derivative of $\Phi$. The first term in this expansion has the form (2) with $F \equiv G \equiv \Phi$ and dependence function

$$
\Omega(x, y)=\varphi\left(\Phi^{-1}(x)\right) \varphi\left(\Phi^{-1}(y)\right), \quad(x, y) \in I^{2}
$$

whose mixed derivative is equal to

$$
\omega(x, y)=\Phi^{-1}(x) \Phi^{-1}(y), \quad(x, y) \in I^{2} .
$$

From (26),

$$
\left(\mu_{\nu}^{\prime}(0) / \sigma_{\nu}(0)\right)^{2}=\left(\mu_{\tilde{\nu}}^{\prime}(0) / \sigma_{\tilde{\nu}}(0)\right)^{2}=0.8537, \quad\left(\mu_{s}^{\prime}(0) / \sigma_{s}(0)\right)^{2}=0.8825
$$

As it follows from [13],

$$
\left(\mu_{r}^{\prime}(0) / \sigma_{r}(0)\right)^{2}=0.6655
$$

and for $p=0.3,0.5,0.7,0.9,1$, respectively,

$$
\begin{aligned}
& \left(\mu_{\tau_{w}}^{\prime}(0) / \sigma_{\tau_{w}}(0)\right)^{2}=0.2736,0.4559,0.6383,0.8207,0.9119 \\
& \left(\mu_{\rho_{w}}^{\prime}(0) / \sigma_{\rho_{w}}(0)\right)^{2}=0.6147,0.7295,0.6271,0.6395,0.9119 .
\end{aligned}
$$

Combining the above results we get Tables 5 and 6 . 
Table 6: Relative efficiencies of the Blest type test statistics versus the weighted Spearman's and Kendall's test statistics for the normal distribution

\begin{tabular}{c|ccccc}
\hline $\begin{array}{c}\text { Test statistics } \\
\text { compared }\end{array}$ & $p=0.3$ & $p=0.5$ & $p=0.7$ & $p=0.9$ & $p=1$ \\
\hline Blest/weighted $\tau$ & 3.12 & 1.87 & 1.34 & 1.04 & 0.94 \\
symmetrized Blest/weighted $\tau$ & 3.23 & 1.94 & 1.38 & 1.08 & 0.97 \\
Blest/weighted $\rho$ & 1.39 & 1.17 & 1.36 & 1.33 & 0.94 \\
symmetrized Blest/weighted $\rho$ & 1.44 & 1.21 & 1.41 & 1.38 & 0.97 \\
\hline
\end{tabular}

Thus, in the normal case, the Blest type statistics are better than $r_{n}$ and the weighted Kendall's and Spearman's coefficients. Also, the efficiency of the symmetrized statistic $s_{n}$ is higher than that of $\nu_{n}$ and $\tilde{\nu}_{n}$.

Example 3. Consider the dependence function

$$
\Omega(x, y)=\min \{x, 1-x\} \min \{y, 1-y\}, \quad(x, y) \in I^{2} .
$$

It arose in studying the conditions of local Bahadur optimality of the Kolmogorov type tests for independence, see [12], Ch. 6. From (25)

$$
\left(\mu_{\nu}^{\prime}(0) / \sigma_{\nu}(0)\right)^{2}=\left(\mu_{\tilde{\nu}}^{\prime}(0) / \sigma_{\tilde{\nu}}(0)\right)^{2}=0.5273, \quad\left(\mu_{s}^{\prime}(0) / \sigma_{s}(0)\right)^{2}=0.5444
$$

From (29)

$$
\left(\mu_{r}^{\prime}(0) / \sigma_{r}(0)\right)^{2}=0.2308
$$

and for $p=0.3,0.5,0.7,0.9,1$, respectively,

$$
\begin{aligned}
& \left(\mu_{\tau_{w}}^{\prime}(0) / \sigma_{\tau_{w}}(0)\right)^{2}=0.1688,0.2813,0.3938,0.5063,0.5625 \\
& \left(\mu_{\rho_{w}}^{\prime}(0) / \sigma_{\rho_{w}}(0)\right)^{2}=0.2956,0.4500,0.4734,0.5169,0.5625 .
\end{aligned}
$$

Combining the above results we arrive at Tables 7 and 8 . 
Table 7: Relative efficiencies of the Blest type test statistics versus the top-down test statistic in Example 3

\begin{tabular}{c|c}
\hline Test statistics compared & Efficiency \\
\hline Blest/top-down & 2.28 \\
symmetrized Blest/top-down & 2.36 \\
\hline
\end{tabular}

Table 8: Relative efficiencies of the Blest type test statistics versus the weighted Spearman's and Kendall's test statistics in Example 3

\begin{tabular}{c|ccccc}
\hline $\begin{array}{c}\text { Test statistics } \\
\text { compared }\end{array}$ & $p=0.3$ & $p=0.5$ & $p=0.7$ & $p=0.9$ & $p=1$ \\
\hline \hline Blest/weighted $\tau$ & 3.12 & 1.87 & 1.34 & 1.04 & 0.94 \\
symmetrized Blest/weighted $\tau$ & 3.23 & 1.94 & 1.38 & 1.08 & 0.97 \\
Blest/weighted $\rho$ & 1.78 & 1.17 & 1.11 & 1.02 & 0.94 \\
symmetrized Blest/weighted $\rho$ & 1.84 & 1.21 & 1.15 & 1.05 & 0.97 \\
\hline
\end{tabular}

Again, the tests based on $\nu_{n}$ and $s_{n}$ have higher efficiency than those based on $r_{n}, \tau_{n, p}$ and $\rho_{n, p}$. At the same time, Kendall's $\tau_{n}$ and Spearman's $\rho_{n}$ remain unbeatable.

In all the examples considered, from the efficiency point of view, the symmetrized statistic $s_{n}$ is better than the original Blest coefficient $\nu_{n}$ and thus to be preferred. 


\section{Chapter 6}

\section{Upper Bound on the Power Function}

Let $\left(X_{1}, Y_{1}\right), \ldots,\left(X_{n}, Y_{n}\right)$ be a random sample from the family of continuous distributions $\left\{\mathbf{P}_{\theta}: \theta \geq 0\right\}$. Denote by $\mathbf{P}_{\theta}^{n}$ the product of $n$ copies of $\mathbf{P}_{\theta}$. Then, having a local parameter $\delta=\sqrt{n} \theta$, we can rewrite $\mathbf{P}_{\theta}^{n}$ as $\mathbf{P}_{\delta / \sqrt{n}}^{n}$ thus getting a statistical model with parameter $\delta$ :

$$
\left(\left(X_{1}, Y_{1}\right), \ldots,\left(X_{n}, Y_{n}\right)\right) \sim\left\{\mathbf{P}_{\delta / \sqrt{n}}^{n}: \delta \geq 0\right\}
$$

Suppose we wish to test the null hypothesis

$$
H_{0}: \delta=0
$$

versus the sequence of alternatives

$$
H_{1}^{n}: \delta>0
$$

using a sequence of test statistics $T_{n}=T_{n}\left(\left(X_{1}, Y_{1}\right), \ldots,\left(X_{n}, Y_{n}\right)\right)$ such that $H_{0}$ is rejected in favor of $H_{1}^{n}$ for large values of $T_{n}$. Let $\pi_{n}(\cdot)$ be the power function of the test based on $T_{n}$. Recall that if the following holds:

$$
\lim _{n \rightarrow \infty} \pi_{n}(\delta / \sqrt{n})=1-\Phi\left(z_{\alpha}-\delta c\right), \quad \delta \geq 0
$$

the test based on $T_{n}$ is said to have slope $c$.

It turns out that under certain regularity conditions on the underlying distribution,

$$
\limsup _{n \rightarrow \infty} \pi_{n}(\delta / \sqrt{n}) \leq 1-\Phi\left(z_{\alpha}-\delta \sqrt{I_{0}}\right), \quad \delta \geq 0
$$


where, for any $\theta \geq 0$,

$$
I_{\theta}=\mathbf{E}_{\theta}\left(\frac{\partial \log h_{\theta}(X, Y)}{\partial \theta}\right)^{2},
$$

is the Fisher information. It follows from (31) and (32) that the square root of the Fisher information $\sqrt{I_{0}}$ is the largest possible slope:

$$
c \leq \sqrt{I_{0}}
$$

The quantity

$$
e:=\frac{c^{2}}{I_{0}}
$$

is the relative efficiency of the test with slope $c$ and the best test. It measures the absolute quality of the test with slope $c$. For this reason, we will call this quantity the Pitman absolute efficiency of the test with slope $c$.

In the next section, we describe the conditions on the sequence of models $\left\{\mathbf{P}_{\theta}^{n}: \theta \geq 0\right\}$ that guarantee the validity of (32).

\subsection{Concept of Local Asymptotic Normality}

Using the notation of the previous section, it can be shown that for large $n$ the models

$$
\left\{\mathbf{P}_{\delta / \sqrt{n}}^{n}: \delta \geq 0\right\} \quad \text { and } \quad\left\{N\left(\delta, I_{0}^{-1}\right): \delta \geq 0\right\}
$$

are similar in statistical properties, whenever the models $\left\{\mathbf{P}_{\theta}^{n}: \theta \geq 0\right\}$ are "smooth" in the parameter. The second model corresponds to the experiment that consists of observing a single observation $Z$ from a normal distribution with mean $\delta$ and variance $I_{0}^{-1}$ equal to the inverse of the Fisher information at $\theta=0$. The condition of local asymptotic normality given below implies convergence of the models $\left\{\mathbf{P}_{\theta}^{n}: \theta \geq 0\right\}$ to a Gaussian model after a rescaling of a parameter. We will formulate the so-called LAN condition only in the case of a real-valued parameter. The general definition that covers the case of a vector-valued parameter can be found, for example, in [8] and [21]. 
Let $H_{\theta}(x, y)$ and $h_{\theta}(x, y)$ be the distribution function and the density corresponding to $\mathbf{P}_{\theta}$. Denote $\mathbf{x}=\left(x_{1}, \ldots, x_{n}\right), \mathbf{y}=\left(y_{1}, \ldots, y_{n}\right), \mathbf{X}=\left(X_{1}, \ldots, X_{n}\right), \mathbf{Y}=\left(Y_{1}, \ldots, Y_{n}\right)$, and put

$$
h_{\theta}(\mathbf{x}, \mathbf{y})=\prod_{i=1}^{n} h_{\theta}\left(x_{i}, y_{i}\right), \quad h_{\theta}(\mathbf{X}, \mathbf{Y})=\prod_{i=1}^{n} h_{\theta}\left(X_{i}, Y_{i}\right)
$$

Definition. The sequence of statistical models $\left\{\mathbf{P}_{\theta}^{n}: \theta \geq 0\right\}$ is called locally asymptotically normal (LAN) at point $\theta=0$, if there exists a sequence $\varphi(n), n=1,2 \ldots$, and a number $I_{0}>0$ such that for any $\delta \geq 0$, the log likelihood ratio process admits the representation

$$
\log \frac{h_{\delta / \varphi(n)}(\mathbf{X}, \mathbf{Y})}{h_{0}(\mathbf{X}, \mathbf{Y})}=\delta \Delta_{n}-\frac{1}{2} I_{0} \delta^{2}+r_{n}(\delta)
$$

where

$$
\Delta_{n} \rightarrow N\left(0, I_{0}\right) \text { in distribution } \mathbf{P}_{0}^{n} \text { as } n \rightarrow \infty \text {, }
$$

and, for any $\delta \geq 0$,

$$
r_{n}(\delta) \rightarrow 0 \text { in probability } \quad \mathbf{P}_{0}^{n} \quad \text { as } \quad n \rightarrow \infty
$$

Now assume that $\left\{\mathbf{P}_{\theta}^{n}: \theta \geq 0\right\}$ is $\operatorname{LAN}$ at $\theta=0$, where $\varphi(n)=\sqrt{n}$, and consider the Gaussian model $\left\{N\left(\delta, I_{0}^{-1}\right): \delta \geq 0\right\}$ with the log likelihood ratio process

$$
\log \frac{d N\left(\delta, I_{0}^{-1}\right)}{d N\left(0, I_{0}^{-1}\right)}(Z)=\delta I_{0} Z-\frac{1}{2} I_{0} \delta^{2} .
$$

The closeness of the two models in (34) is now easily seen from the similarity of the quadratic expansions in (35) and (36).

It is well known, see [21], Theorem 15.4, that if the family of models $\left\{\mathbf{P}_{\theta}^{n}: \theta \geq 0\right\}$ is LAN at $\theta=0$, then the upper bound (32) holds. In the next section, we will show that if $H_{\theta}(x, y)$ belongs to the class $\mathcal{H}$, then the LAN condition is fulfilled at point $\theta=0$ with the normalizing factor $\varphi(n)=\sqrt{n}$. 


\subsection{LAN of the Underlying Family of Distributions}

The local asymptotic normality of the family at hand is easily verified by means of Hájek's theorem, see, for example, [8], Section 2.2. According to this theorem, we only have to check that: (1) the density $h_{\theta}(x, y)$ is absolutely continuous in $\theta$ in some neighborhood of $\theta=0$ for all $(x, y) \in I^{2} ;(2)$ the derivative $\partial h_{\theta}(x, y) / \partial \theta$ exists for every $\theta$ belonging to this neighborhood for $\lambda$-almost all $(x, y) \in I^{2}$; (3) the function $I_{\theta}$ is continuous and positive for $\theta=0$.

The Fisher information corresponding to $H_{\theta} \in \mathcal{H}$ is equal to

$$
I_{\theta}=\iint \frac{\omega^{2}(x, y)}{1+\theta \omega(x, y)} d x d y \text {. }
$$

Therefore, under the assumption that the underlying d.f. $H_{\theta}$ belongs to $\mathcal{H}$, conditions (1)-(3) are trivially satisfied.

\subsection{Pitman Absolute Efficiency of the Blest Type Tests}

Now we obtain the formulas for the Pitman absolute efficiency of the tests under consideration. Combining Lemmas 1-3 with (33) we arrive at the following upper bounds on the limiting power functions of the Blest type tests:

$$
\left(\frac{\mu_{\nu}^{\prime}(0)}{\sigma_{\nu}(0)}\right)^{2} \leq I_{0}, \quad\left(\frac{\mu_{\tilde{\nu}}^{\prime}(0)}{\sigma_{\tilde{\nu}}(0)}\right)^{2} \leq I_{0}, \quad\left(\frac{\mu_{s}^{\prime}(0)}{\sigma_{s}(0)}\right)^{2} \leq I_{0}
$$

where

$$
I_{0}=\iint_{I^{2}} \omega^{2}(x, y) d x d y
$$

Therefore, recalling (25), we get the following expressions for the Pitman absolute efficiency of the test statistics $\nu_{n}, \tilde{\nu}_{n}$ and $s_{n}$ :

$$
\begin{aligned}
e_{\nu} & =135 \frac{\left(\iint_{I^{2}}(1-x)^{2} y \omega(x, y) d x d y\right)^{2}}{\iint_{I^{2}} \omega^{2}(x, y) d x d y} \\
e_{\tilde{\nu}} & =135 \frac{\left(\iint_{I^{2}} x(1-y)^{2} \omega(x, y) d x d y\right)^{2}}{\iint_{I^{2}} \omega^{2}(x, y) d x d y} \\
e_{s} & =\frac{1080\left(\iint_{I^{2}} x y(4-x-y) \omega(x, y) d x d y\right)^{2}}{31 \iint_{I^{2}} \omega^{2}(x, y) d x d y}
\end{aligned}
$$




\section{Chapter 7}

\section{Local Asymptotic Optimality of the Blest Type}

\section{Tests}

The problem of finding the structure of a distribution for which a given nonparametric statistic is optimal is an important and worthwhile problem, since the overwhelming majority of nonparametric statistics have been proposed for empirical reasons. In the opinion of their authors these statistics "must work" in one or another problem of testing statistical hypotheses. The problem of determining the "best" alternative has been studied by several authors, see [12], Sect. 6.1, for references. Below, we will show that for a special structure of the dependence function $\Omega$, the statistics $\nu_{n}, \tilde{\nu}_{n}$, and $s_{n}$ possesses the Pitman optimality property.

Due to the upper bounds (37), the problem of determining the "optimal" distribution function can be reduced to the problem of minimization of the functional $I_{0}=\iint_{I^{2}} \omega^{2} d x d y$ subject to certain boundary and normalization conditions.

Theorem 1. The sequence of test statistics $\left\{\nu_{n}\right\}$ is Pitman optimal if and only if the dependence function has the form

$$
\Omega(x, y)=C x(1-x)(2-x) y(1-y), \quad(x, y) \in I^{2}, \quad C>0 .
$$

By the symmetry, the following result also holds.

Theorem 2. The sequence of test statistics $\left\{\tilde{\nu}_{n}\right\}$ is Pitman optimal if and only if the 
dependence function has the form

$$
\Omega(x, y)=C x(1-x) y(1-y)(2-y), \quad(x, y) \in I^{2}, \quad C>0 .
$$

Proof of Theorem 1. According to (25) and (38), the upper bound corresponding to $\nu_{n}$ takes the form

$$
135\left(\iint_{I^{2}}(1-x)^{2} y \Omega_{x y}(x, y) d x d y\right)^{2} \leq \iint_{I^{2}} \Omega_{x y}^{2}(x, y) d x d y .
$$

Thus we need to find the infimum of the functional $J(\Omega)=\iint_{I^{2}} \Omega_{x y}^{2}(x, y) d x d y$ over the space $\mathbf{C}_{0}^{2}\left(I^{2}\right)$ under the normalization condition

$$
J_{1}(\Omega)=\iint_{I^{2}}(1-x)^{2} y \Omega_{x y}(x, y) d x d y=1
$$

and the boundary condition

$$
\Omega\left(\partial I^{2}\right)=0
$$

This problem is a typical isoperimetric problem that consists in finding an optimum of a certain functional, say, $J(f)$, over a functional class $\mathcal{F}=\{f\}$ subject to the condition $J_{1}(f)=$ const., for some other functional $J_{1}(f)$. Due to the Lagrange principle, the above isoperimetric problem (see, for example, [4]) can be reduced to the problem of finding the infimum of the functional

$$
K(\Omega)=J(\Omega)+\lambda J_{1}(\Omega)
$$

where $\lambda$ is the Lagrange multiplier. Let $a(x, y)$ be an admissible function, i.e., $a \in \mathbf{C}_{0}^{2}\left(I^{2}\right)$. A necessary condition for $K(\Omega)$ to have an extremum for $\Omega=\hat{\Omega}$ is that its variation vanishes for $\Omega=\hat{\Omega}$, i.e.,

$$
\left.\frac{d}{d t} K(\hat{\Omega}+t a)\right|_{t=0}=0
$$

Equality (40) can be written as

$$
\begin{aligned}
\frac{d}{d t} \iint_{I^{2}}\left\{\left(\frac{\partial^{2}}{\partial x \partial y}(\hat{\Omega}(x, y)+t a(x, y))\right)^{2}\right. & \\
& \left.+\lambda(1-x)^{2} y \frac{\partial^{2}}{\partial x \partial y}(\hat{\Omega}(x, y)+t a(x, y))\right\}\left.d x d y\right|_{t=0}=0,
\end{aligned}
$$


or equivalently,

$$
\iint_{I^{2}}\left(2 \hat{\Omega}_{x y}(x, y)+\lambda(1-x)^{2} y\right) a_{x y}(x, y) d x d y=0
$$

Using the integration by parts technique, together with the boundary condition $a\left(\partial I^{2}\right)=0$, we obtain

$$
\iint_{I^{2}}\left(2 \hat{\Omega}_{x x y y}(x, y)-2 \lambda(1-x)\right) a(x, y) d x d y=0 .
$$

Then, applying the Lemma on page 22 in [4], we arrive at the boundary-value problem

$$
\left\{\begin{array}{l}
\hat{\Omega}_{x x y y}(x, y)=C(1-x), \\
\hat{\Omega}\left(\partial I^{2}\right)=0
\end{array}\right.
$$

with some constant $C$. This is equivalent to the integral equation, see, for example, [23], Section 2.7,

$$
\hat{\Omega}(x, y)=C \iint_{I^{2}} \mathcal{G}(x, y ; s, t)(1-s) d s d t
$$

where $\mathcal{G}$ is the Green function given by

$$
\mathcal{G}(x, y ; s, t)=(\min \{x, s\}-x s)(\min \{y, t\}-y t), \quad 0 \leq x, y, s, t \leq 1 .
$$

Integration on the right-hand side of (41) yields

$$
\begin{aligned}
\hat{\Omega}(x, y) & =\int_{y}^{1} \int_{0}^{x}(x-x s)(t-y t)(1-s) d s d t \\
& +\int_{0}^{y} \int_{x}^{1}(x-x s)(t-y t)(1-s) d s d t \\
& +\int_{0}^{y} \int_{0}^{x}(s-x s)(t-y t)(1-s) d s d t \\
& +\int_{y}^{1} \int_{x}^{1}(x-x s)(y-y t)(1-s) d s d t \\
& =\frac{1}{12} x y(x-1)(y-1)(2-x)
\end{aligned}
$$

and

$$
\hat{\omega}(x, y)=\hat{\Omega}_{x y}(x, y)=-\frac{1}{12}\left(2-6 x+3 x^{2}\right)(2 y-1) .
$$


It is easy to check that the optimum obtained is indeed a minimum. It remains to show that the optimum is unique. By the Cauchy-Schwarz inequality, equality in (39) holds if and only if $\Omega_{x y}(x, y)$ is proportional to $(1-x)^{2} y$ almost everywhere. Note that the boundary condition $\Omega\left(\partial I^{2}\right)=0$ implies

$$
\iint_{I^{2}} x^{k} \Omega_{x y}(x, y) d x d y=\iint_{I^{2}} y^{k} \Omega_{x y}(x, y) d x d y=0, \quad k=0,1,2, \ldots
$$

From this, we may immediately conclude that $\hat{\omega}(x, y)=\hat{\Omega}_{x y}(x, y)$ is proportional to $(1-x)^{2} y$ almost everywhere. The proof is completed.

Theorem 3. The sequence of test statistics $\left\{s_{n}\right\}$ is Pitman optimal if and only if the dependence function has the form

$$
\Omega(x, y)=C x(1-x) y(1-y)(4-x-y), \quad(x, y) \in I^{2}, \quad C>0 .
$$

Proof. The proof of Theorem 3 is similar to that of Theorem 1. The upper bound corresponding to $s_{n}$ takes the form

$$
1080\left(\iint_{I^{2}} x y(4-x-y) \Omega_{x y}(x, y) d x d y\right)^{2} \leq \iint_{I^{2}} \Omega_{x y}^{2}(x, y) d x d y .
$$

Here, we consider the minimization problem of the functional $J(\Omega)=\iint_{I^{2}} \Omega_{x y}^{2}(x, y) d x d y$ over the space $\mathrm{C}_{0}^{2}\left(I^{2}\right)$ subject to the normalization condition

$$
J_{1}(\Omega)=\iint_{I^{2}} x y(4-x-y) \Omega_{x y}(x, y) d x d y=1,
$$

and the boundary condition

$$
\Omega\left(\partial I^{2}\right)=0
$$

Application of the Lagrange principle leads to the boundary-value problem

$$
\left\{\begin{array}{l}
\hat{\Omega}_{x x y y}(x, y)=C(2-x-y), \\
\hat{\Omega}\left(\partial I^{2}\right)=0
\end{array}\right.
$$

whose solution is given by

$$
\hat{\Omega}(x, y)=C \iint_{I^{2}} \mathcal{G}(x, y ; s, t)(2-s-t) d s d t
$$


with the Green function $\mathcal{G}$ as in (41). By integrating on the right-hand side of (45), we obtain

$$
\begin{aligned}
\hat{\Omega}(x, y) & =\int_{y}^{1} \int_{0}^{x}(x-x s)(t-y t)(2-s-t) d s d t \\
& +\int_{0}^{y} \int_{x}^{1}(x-x s)(t-y t)(2-s-t) d s d t \\
& +\int_{0}^{y} \int_{0}^{x}(s-x s)(t-y t)(2-s-t) d s d t \\
& +\int_{y}^{1} \int_{x}^{1}(x-x s)(y-y t)(2-s-t) d s d t \\
& =\frac{1}{12} x y(x-1)(y-1)(4-x-y),
\end{aligned}
$$

and hence

$$
\hat{\omega}(x, y)=\hat{\Omega}_{x y}(x, y)=\frac{1}{3}-\frac{5}{6}(x+y)+\frac{1}{4} x^{2}-\frac{1}{2} x^{2} y+\frac{1}{4} y^{2}-\frac{1}{2} x y^{2}+2 x y .
$$

Again, due to the Cauch-Schwarz inequality and (42), the function $\hat{\omega}(x, y)=\hat{\Omega}_{x y}(x, y)$ is proportional to $x y(4-x-y)$ almost everywhere. Thus, equality in (44) is attained if and only if $\Omega(x, y)$ is given by $(43)$. 


\section{Chapter 8}

\section{Conclusion}

In this project, based on the concept of asymptotic relative efficiency (ARE), we calculate asymptotic efficiency of the Blest type correlation coefficients for a wide class of alternatives close to the null hypothesis of independence. We also compare them with the "top-down" correlation coefficient, Spearman's $\rho$, Kendall's $\tau$ and their weighted versions. It is shown that, from the efficiency point of view, the symmetrized statistics $s_{n}$ is better than the original Blest coefficient $\nu_{n}$, and that the Blest type tests have higher efficiency than the "top-down" correlation coefficient. Moreover, we find the structures of alternatives for which tests at hand are asymptotically optimal in the sense of Pitman. Our results complete the efficiency study in Genest and Plante [5] where the problem of determining conditions under which the tests based on $\nu_{n}, \tilde{\nu}_{n}$ and $s_{n}$ are asymptotically optimal was touched but remained unsolved. 


\section{Appendix A}

\section{Calculation of Relative Efficiency}

I Calculation of relative efficiency on pages 30-31 in Matlab.

1. Blest versus top-down for Woodworth distribution

syms $\mathrm{x}$ y $\mathrm{k}$

$01=\mathrm{x} *\left(1-\mathrm{x}^{\wedge} \mathrm{k}\right) * \mathrm{y} *\left(1-\mathrm{y}^{\wedge} \mathrm{k}\right)$;

$02=\operatorname{subs}(01, \mathrm{k}, 10)$;

omega $=02$;

$\mathrm{f}=(1-\mathrm{x}) *$ omega ;

$f 1=\operatorname{int}(f, x, 0,1)$;

$f 2=\operatorname{int}(f 1, y, 0,1)$;

$f 3=$ omega $/(x * y)$;

$f 4=\operatorname{int}(f 3, x, 0,1)$;

$f 5=\operatorname{int}(f 4, y, 0,1)$;

$e 1=540 *(f 2 / f 5)^{-} 2$;

$e=$ double $(e 1) ; e$

2. Symmetrized Blest versus top-down for Woodworth distribution syms $x$ y $k$

$01=\mathrm{x} * \mathrm{y} *\left(1-\mathrm{x}^{\wedge} \mathrm{k}\right) *\left(1-\mathrm{y}^{\wedge} \mathrm{k}\right)$;

$02=\operatorname{subs}(01, \mathrm{k}, 10)$;

omega $=02$; 


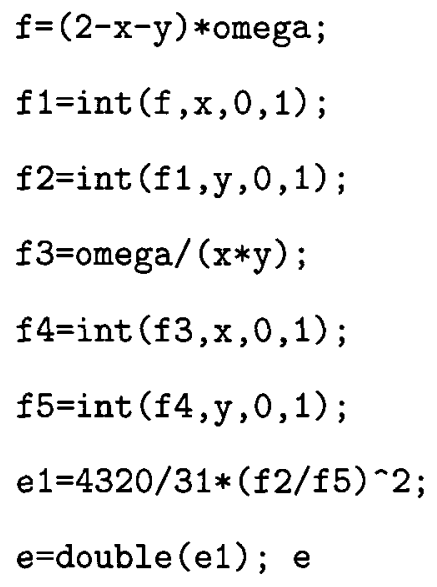

3. Symmetrized Blest versus weighted Kendall's for Woodworth distribution

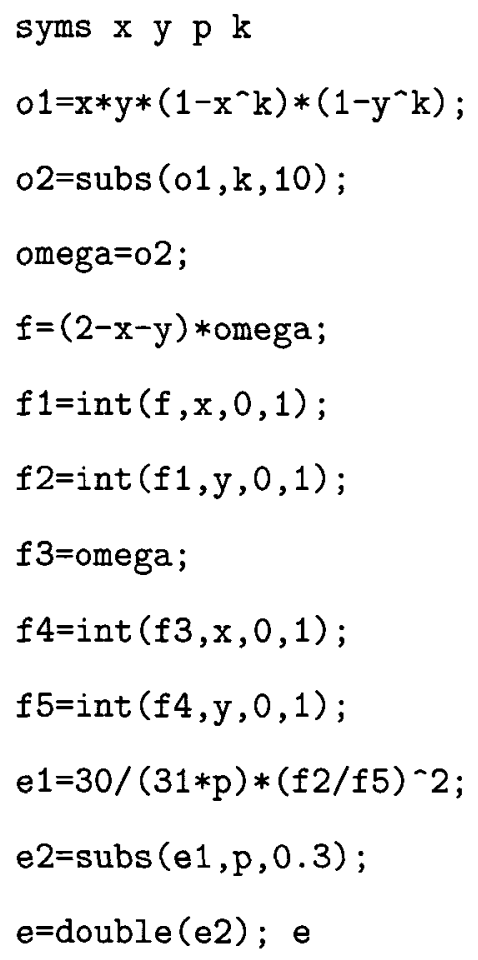

4. Symmetrized Blest versus weighted Spearman's for Woodworth distrbution 


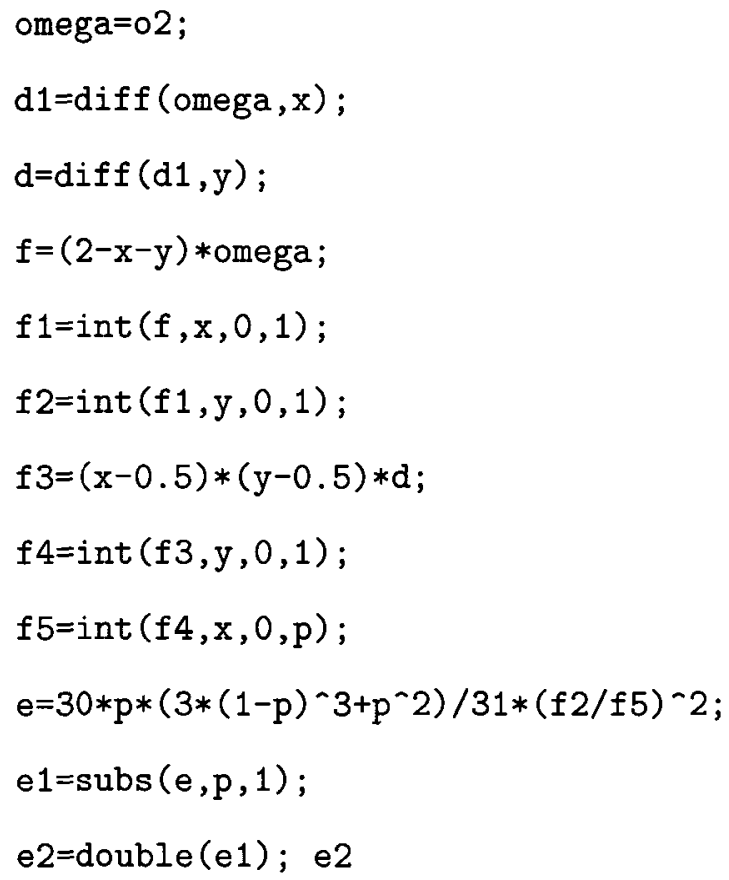

5. Blest versus Kendall's for Woodworth distribution

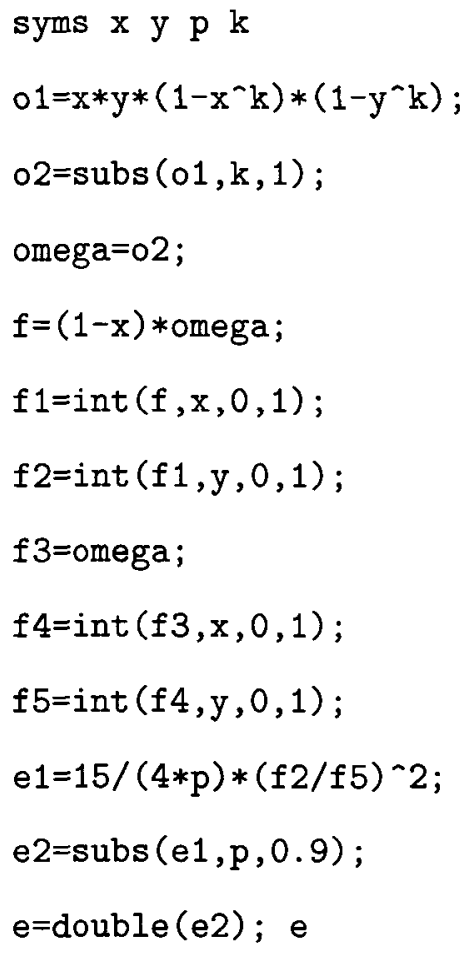

6. Blest versus Spearman's for Woodworth distribution syms $x$ y $p$ k 


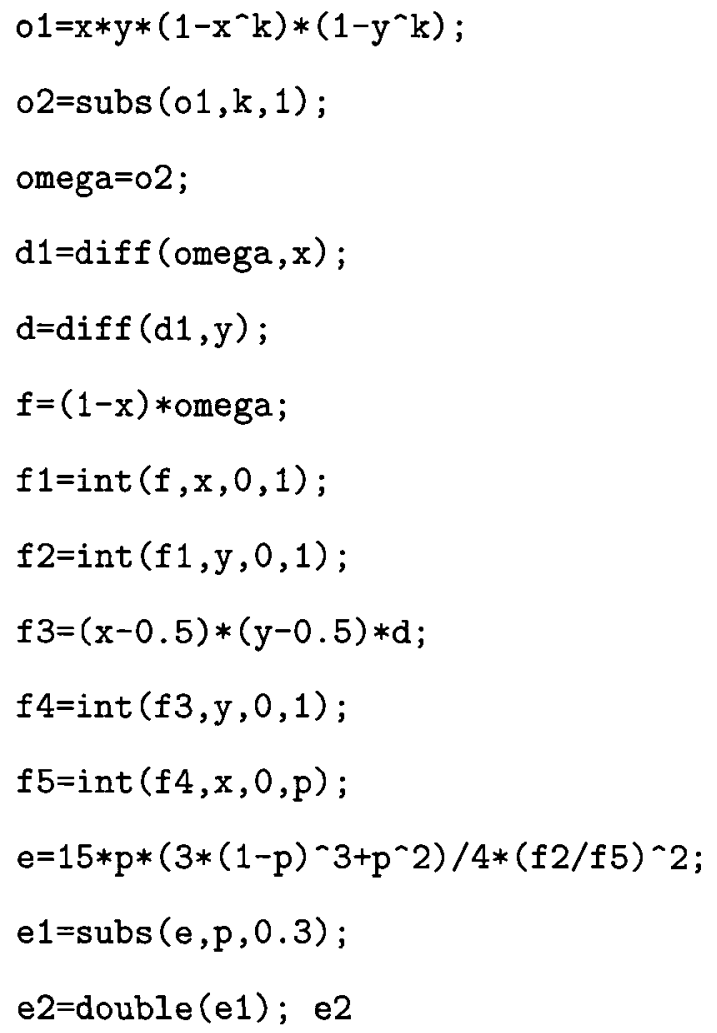

II Calculation in Maple for bivariate normal case on page 33.

1. Relative efficiency of the Blest's test statistics with (stats); $a:=-10 ; b:=10 ; n:=1000 ; t:=(b-a) / n ; s:=0 ;$ for $i$ from 1 to $\mathrm{n}$ do $s:=s+t *($ statevalf $[p d f, \operatorname{normald}](x)) \leadsto 2 /$ statevalf [cdf, normald] (x); $\mathrm{x}:=\mathrm{a}+\mathrm{t} * \mathrm{i} ;$ end do; $\mathrm{s}$;

with (stats); $a:=-20 ; b:=20 ; n:=1000 ; t:=(b-a) / n ; s:=0 ;$ for $i$ from 1 to $n$ do $s:=s+t * s t a t e v a l f[p d f$, normald $](x) *(1-\operatorname{statevalf}[\operatorname{cdf}$, normald $](x)) \wedge 2 * x$; $x:=a+t * i ;$ end do; $s$;

2. Relative efficiency of the symmetrized Blest with (stats); $a:=-5 ; b:=5 ; n:=1000 ; t:=(b-a) / n ; s:=0 ;$ for $i$ 
from 1 to $n$ do

$\mathrm{s}:=\mathrm{s}+\mathrm{t} *\left(\right.$ statevalf $[\mathrm{pdf}, \text { normald] }(\mathrm{x}))^{\wedge} 2 *\left(4 *\left(\right.\right.$ statevalf $[\mathrm{cdf}, \text { normald] }(\mathrm{x}))^{\wedge} 2$

$\left.-2 *(\text { statevalf [cdf, normald] }(\mathrm{x}))^{\wedge} 3\right) * \mathrm{x}^{\wedge} 2 ; \mathrm{x}:=\mathrm{a}+\mathrm{t} * \mathrm{i} ;$ end do; $\mathrm{s}$; 


\section{List of References}

[1] D. C. Blest (2000). Rank correlation - an alternative measure. Australian and New Zealand Journal of Statistics, 42, 101-111.

[2] H. Cramer (1946). Mathematical Methods of Statistics. Princeton Univ. Press.

[3] D. J. G. Farlie (1960). The performance of some correlation coefficients for a general bivariate distribution. Biometrika, 47, 307-323.

[4] I. M. Gelfand and S. V. Fomin (1963). Calculus of Variations. Prentice-Hall, Inc.

[5] C. Genest and J.-F. Plante (2003). On Blest's measure of rank correlation. The Canadian Journal of Statistics, 31, No. 1, 1-18.

[6] J. Hájek and Z. Sidák (1967). Theory of Rank Tests. Academic Press, New York.

[7] T. P. Hettmansperger (1984). Statistical Inference Based on Ranks. Wiley.

[8] I. A. Ibragimov and R. Z. Has'minskii (1981). Statistical Estimation: Asymptotic Theory. Springer, New York.

[9] R. L. Iman and W. J. Conover (1987). A measure of top-down correlation. Technometrics, 29, 351-357.

[10] S.C. Kochar and R. P. Gupta (1987). Competitors of the Kendall's tau test for testing independence against positive quadrant dependence. Biometrika, 74, 664-666.

[11] M. G. Kendall (1938). A new measure of rank correlation. Biometrika, 30, 81-93.

[12] Ya. Yu. Nikitin (1995). Asymptotic Efficiency of Nonparametric Tests. Cambridge Univ. Press.

[13] Ya. Yu. Nikitin and N. A. Stepanova (2000). A generalization of Kendalls' tau and the asymptotic efficiency of the corresponding independence tests. Journal of Mathematical Sciences, 99, No. 6, 1154-1160.

[14] Ya. Yu. Nikitin, N. A. Stepanova (2003). Pitman efficiency of independence tests based on weighted rank statistics. Journal of Mathematical Sciences, 118, No. 6, 5596-5606. 
[15] F. H. Ruymgaart, G. R. Shorack, W. R. van Zwet (1972). Asymptotic normality of nonparametric tests for independence. The Annals of Mathematical Statistics, 43, $1122-1135$.

[16] F. H. Ruymgaart (1978). Asymptotic Theory of Rank Tests of Independence. Mathematical Centre Tracts, 43, Amsterdam.

[17] M. Scarsini (1984). On measures of concordance. Stochastica, 8, 201-218.

[18] R. J. Serfling (1980). Approximation Theorems of Mathematical Statistics. Wiley, New York.

[19] G. S. Shieh, Z. Bai, and W.-Y. Tsai (2000). Rank tests of independence with a weighted contanimation alternative. Statist. Sinica, 10, 577-594.

[20] C. Spearman (1904). The proof and measurement of association between two things. American Jourmal of Psychology, 15, 72-101.

[21] A. W. van der Vaart (2000). Asymptotic Statistics. Cambridge Univ. Press.

[22] G. G. Woodworth (1966). On the asymptotic theory of tests of independence based on bivariate layer ranks. Dept. of Statistics, Univ. of Minnesota. Tech. Report No. 75.

[23] E. Zeidler (1995). Applied Functional Analysis: Applications to Mathematical Physics. Springer. 